\title{
The Highly Regioselective Synthesis of Novel Imidazolidin-2- Ones via the Intramolecular Cyclization/Electrophilic Substitution of Urea Derivatives and the Evaluation of Their Anticancer Activity
}

\author{
Almir S. Gazizov ${ }^{1, *(\mathbb{D})}$, Andrey V. Smolobochkin ${ }^{1}{ }^{(D)}$, Elizaveta A. Kuznetsova ${ }^{1}$, Dinara S. Abdullaeva ${ }^{2}$, \\ Alexander R. Burilov ${ }^{1}$, Michail A. Pudovik ${ }^{1}$, Alexandra D. Voloshina ${ }^{1}$, Victor V. Syakaev ${ }^{1}$, Anna P. Lyubina ${ }^{1}$, \\ Syumbelya K. Amerhanova ${ }^{1}$ and Julia K. Voronina ${ }^{3,4}$ (D)
}

check for

updates

Citation: Gazizov, A.S.;

Smolobochkin, A.V.; Kuznetsova,

E.A.; Abdullaeva, D.S.; Burilov, A.R.;

Pudovik, M.A.; Voloshina, A.D.;

Syakaev, V.V.; Lyubina, A.P.;

Amerhanova, S.K.; et al. The Highly

Regioselective Synthesis of Novel

Imidazolidin-2-Ones via the

Intramolecular Cyclization/

Electrophilic Substitution of Urea

Derivatives and the Evaluation of

Their Anticancer Activity. Molecules

2021, 26, 4432 .

https://doi.org/10.3390/

molecules26154432

Academic Editor: Jacek Nycz

Received: 29 June 2021

Accepted: 20 July 2021

Published: 22 July 2021

Publisher's Note: MDPI stays neutral with regard to jurisdictional claims in published maps and institutional affiliations.

Copyright: (c) 2021 by the authors. Licensee MDPI, Basel, Switzerland. This article is an open access article distributed under the terms and conditions of the Creative Commons Attribution (CC BY) license (https:/ / creativecommons.org/licenses/by/ $4.0 /)$.
1 Arbuzov Institute of Organic and Physical Chemistry, FRC Kazan Scientific Center, Russian Academy of Sciences, Arbuzova Str. 8, 420088 Kazan, Russia; smolobochkin@iopc.ru (A.V.S.); sherlok@live.ru (E.A.K.); burilov@iopc.ru (A.R.B.); pudovik@iopc.ru (M.A.P.); microbi@iopc.ru (A.D.V.); vvs@iopc.ru (V.V.S.); anna.1@mail.ru (A.P.L.); s.amerkhanova@mail.ru (S.K.A.)

2 Chemistry Department, Kazan Federal University, Kremlyovskaya Str. 18, 420008 Kazan, Russia; dinka0902@mail.ru

3 N.S. Kurnakov Institute of General and Inorganic Chemistry, Russian Academy of Sciences, Leninsky Ave. 31, 119991 Moscow, Russia; juliavoronina@mail.ru

4 G.V. Plekhanov Russian University of Economics, Stremyanny Per. 36, 117997 Moscow, Russia

* Correspondence: agazizov@iopc.ru

Abstract: A series of novel 4-(het)arylimidazoldin-2-ones were obtained by the acid-catalyzed reaction of (2,2-diethoxyethyl)ureas with aromatic and heterocyclic $C$-nucleophiles. The proposed approach to substituted imidazolidinones benefits from excellent regioselectivity, readily available starting materials and a simple procedure. The regioselectivity of the reaction was rationalized by quantum chemistry calculations and control experiments. The anti-cancer activity of the obtained compounds was tested in vitro.

Keywords: imidazolidine-2-one; regioselectivity; urea; cyclization; anti-tumor activity; anti-cancer activity; cytotoxicity

\section{Introduction}

Various cyclic ureas are the well-established pivotal functionalities in the drug discovery and medicinal chemistry. Among them, the imidazolidine-2-one scaffold is often found in bioactive compounds and constitute the key structural part of a plethora of FDA approved drugs, such as emicerfont, imidapril, azlocillin and others (Figure 1). Moreover, these compounds may serve as precursors for vicinal diamines, which are valuable building blocks in organic and medicinal chemistry [1,2]. Taking these into account, it is not surprising that a lot of effort is devoted to the development of the methods of synthesis of imidazolidinones [3].

The most straightforward approach to cyclic ureas is the carbonylation of variously substituted diamines. This approach is somewhat controversial due to the abovementioned utility of imidazolidine-2-ones as 1,2-diamines precursors. However, it is still used in some cases, especially with chiral diamines [4-7]. Novel approaches to these compounds have been developed in 2019-2020 by various research groups, indicating a growing area of interest. These include silver-catalyzed cycloaddition of nitrones with methylene isocyanides [8], stereoselective diamination of alkenes with 1,3-ditosylurea [9] and Rh-catalyzed intramolecular C-H amination of $N$-oxyurea derivatives $[10,11]$. Pdcatalyzed amidation of vinylethylcarbamates followed by cyclization of intermediate 
allyl ureas should also be mentioned [12]. Finally, the amidoalkylation of indoles by hydroxyimidazolidin-2-one under the Lewis acid catalysis has also been reported [13].

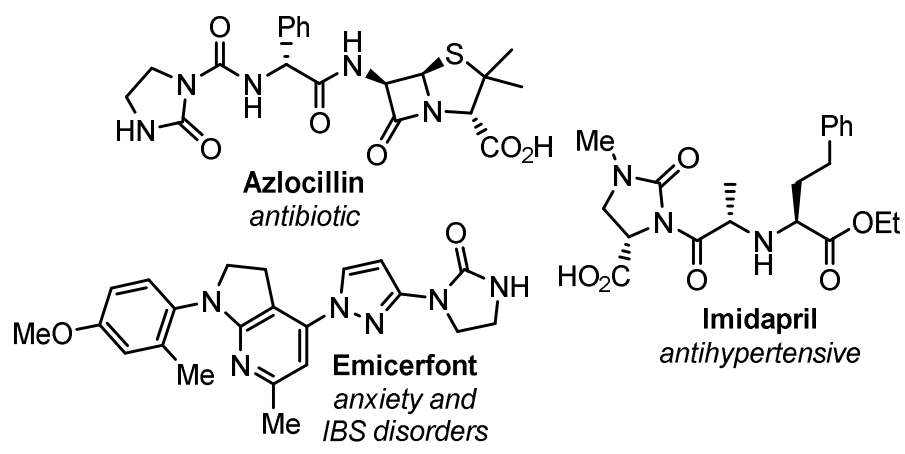

Figure 1. Some known drugs possessing imidazolidin-2-one moiety.

Nitrogen-containing aldehydes and acetals are widely used in the heterocycles synthesis $[14,15]$. Earlier, we have described the approach to 2-substituted pyrrolidine derivatives based on the in situ generation of a cyclic iminium ion from 4-aminobutanal acetals (4,4diethoxybutan-1-amines) and its trapping by various nucleophiles [16-18]. Our preliminary studies indicate that $\mathrm{N}$-(2,2-dialkoxyethyl) ureas are also capable of the formation of cyclic imidazolinium cations and the reaction results in cyclic urea derivatives-imidazolidinones or benzo $[d][1,3]$ diazepinones [19]. As the continuation of our efforts, herein, we report the successful application of this methodology to the synthesis of the series of 4-(hetero) arylimidazolidin-2-ones starting from $\mathrm{N}$-(2,2-dialkoxyethyl) ureas and (hetero) aromatic nucleophiles (Scheme 1). The main advantages of the described method are the mild reaction conditions, good to high product yield and the high regioselectivity. Notably, N-(2,2dialkoxyethyl) ureas are easily accessible from commercially available 2,2-dimethoxyethan1-amine and amines or isocyanates in just one step, which also adds value to the proposed approach. Some considerations on the regioselectivity of the reaction are discussed based on quantum chemistry calculations, as well as evaluation of the in vitro anti-cancer activities of the obtained compounds.

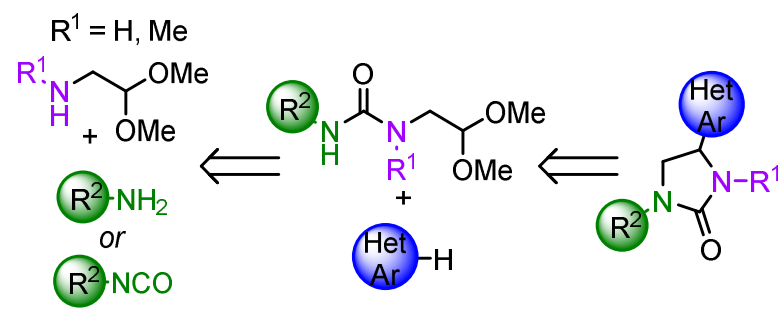

Scheme 1. The proposed synthetic approach to 4-(hetero) arylimidazolidine-2-ones.

\section{Results and Discussion}

\subsection{Chemistry}

We initiated our studies with the expanding the scope of the starting $N$-(2,2-dialkoxyethyl) ureas 1 (Scheme 2). Di- and trisubstituted ureas possessing various aryl (1a-f) and aryl/alkyl $(\mathbf{g} \mathbf{-} \mathbf{m})$ groups were easily obtained from appropriate isocyanates by known procedures [20].

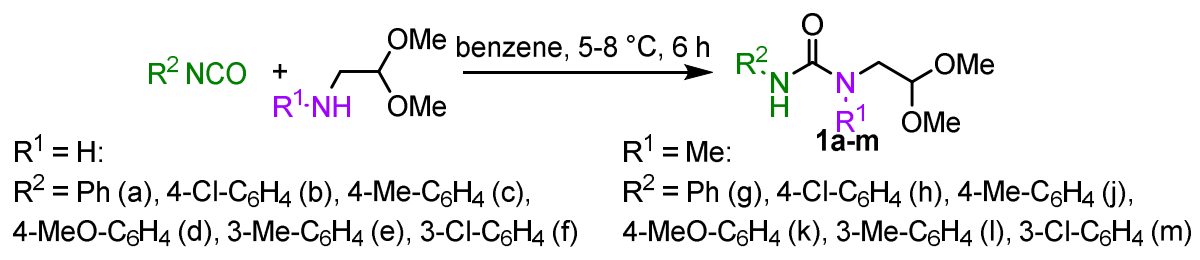

Scheme 2. The synthesis of starting N-(2,2-dialkoxyethyl)ureas 1. 
Next, we investigated the reaction of the ureas 1a-m with various electron-rich aromatic and heterocyclic $C$-nucleophiles (Scheme 3 ). In all cases, the reaction provided the desired imidazolidinones $\mathbf{2}$ in good to high yield. The previously reported conditions were employed, i.e., refluxing toluene and a triflouroacetic acid (TFA) as catalysts [19]. Notably, the 15-fold excess of catalyst was used in these preliminary experiments. However, the further screening of reaction conditions allowed us to decrease the amount of catalyst significantly without a loss of yield. Moreover, during our preliminary studies, we found that this reaction leads to the mixture of 4- and 5-substituted imidazolidin-2-ones, with 4-substituted regioisomer 2 being the major one. Decreasing the amount of TFA led to the improved regioselectivity, so that only 4-substituted imidazolinones 2 were observed in the reaction mixture. The isolated yields of target compounds varied somewhat. In general, ureas possessing unsubstituted nitrogen atom tend to provide higher yields of imidazolidinones 2 . However, no correlation was observed between the yields and the substituents in the aryl moiety of the starting ureas.

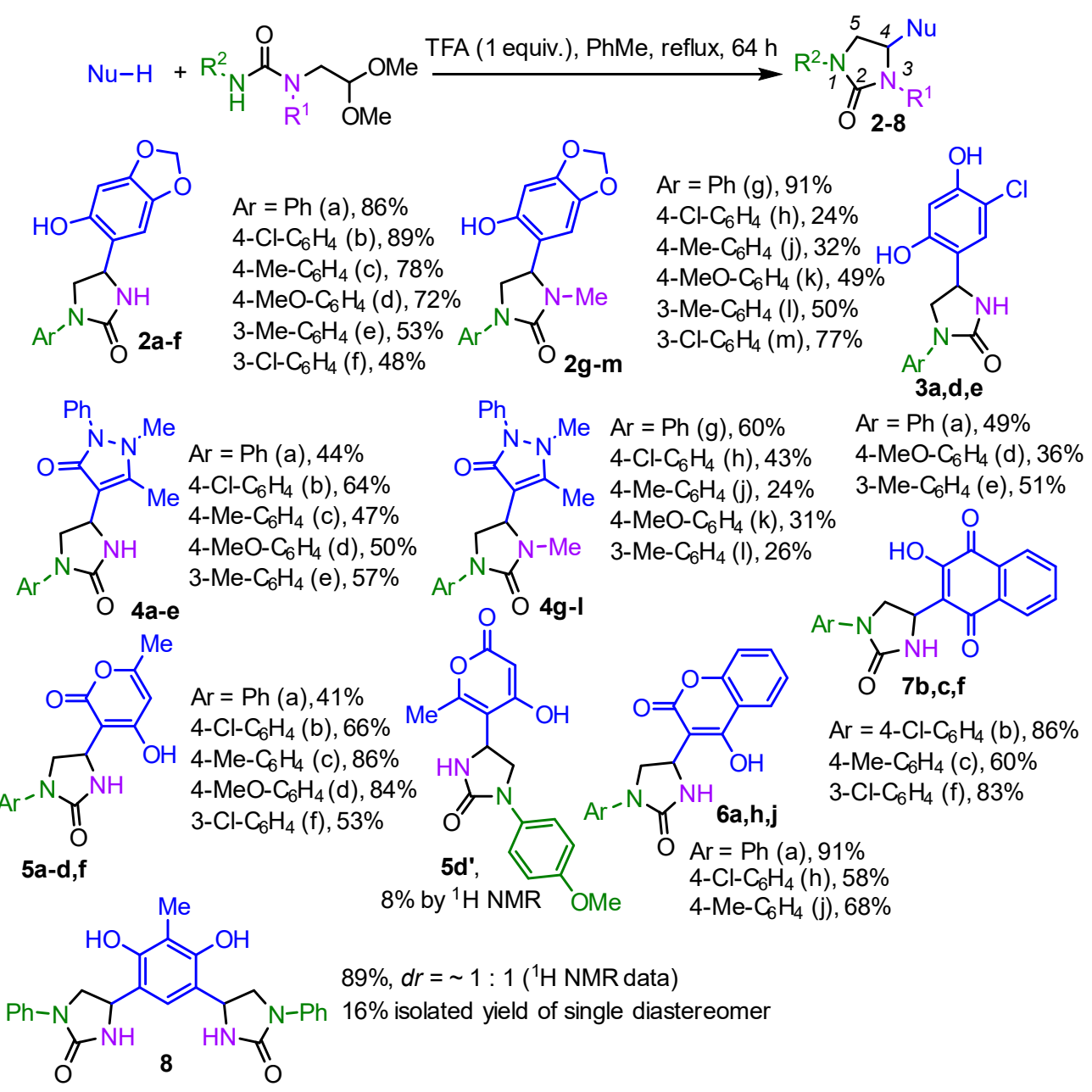

Scheme 3. The synthesis of imidazolidine-2-ones 2. The isolated yields are given, unless noted otherwise.

The chemical shifts of methyne and methylene protons for 4- and 5-substituted imidazolidin-2-ones differ notably [19] (see Supplementary Materials, Figure S2). Thus, the substitution site of the imidazolidinone ring for the $\mathrm{N}$-methyl substituted compounds $2 \mathrm{~h}-\mathrm{m}$ and $4 \mathrm{~h}-1$ was determined by the comparison of their NMR spectra with the spectra of previously described 4-substituted imidazolidin-2-ones, $\mathbf{2 g}$ and $\mathbf{4 g}$ [19] (for example, see Supplementary Materials, Figures S3 and S4). Additionally, the structures of the compounds $\mathbf{2} \mathbf{j}$ and $\mathbf{2 k}$ were confirmed by $\mathrm{X}$-ray analysis (Figure 2; for details, see Supplementary Materials, pp. S2-S3 and Figure S1). 


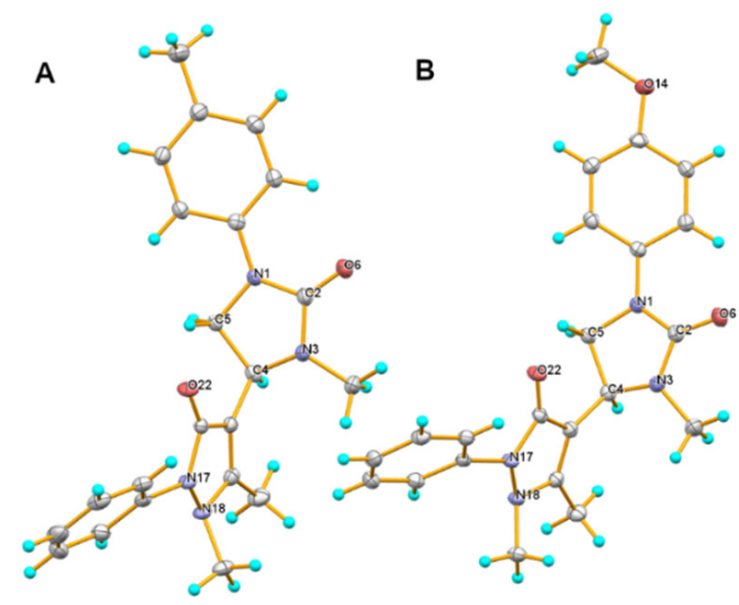

Figure 2. Molecular structure of the compounds $2 \mathbf{j}(\mathbf{A})$ and $2 \mathbf{k}(\mathbf{B})$. Ellipsoids are given with a 50\% probability.

In order to determine the substitution pattern on imidazolidine-2-one ring correctly for other compounds, a complete structure elucidation of the compound $2 \mathrm{c}$ was accomplished by a variety of $1 \mathrm{D} / 2 \mathrm{D}$ NMR correlation experiments $\left({ }^{1} \mathrm{H}-{ }^{1} \mathrm{H}\right.$ COSY, NOESY, ${ }^{1} \mathrm{H}-{ }^{13} \mathrm{C}$ HSQC, ${ }^{1} \mathrm{H}^{13} \mathrm{C} \mathrm{HMBC},{ }^{1} \mathrm{H}-{ }^{15} \mathrm{~N}$ HMBC). First, the signals of protons and carbon atoms were assigned used the set of 2D homo- and heteronuclear correlation experiments. Then, the 1D NOESY experiment was carried out. Selective excitation of ortho-protons $\left(\mathrm{H}^{\mathrm{a}}\right)$ of aryl fragment provided NOE between these protons and $\mathrm{H}^{\mathrm{b}}, \mathrm{H}^{\mathrm{b}}$ protons of the methylene group $\left(\mathrm{H}^{\mathrm{b}} 2.0 \%, \mathrm{H}^{\mathrm{b}} 2.1 \%\right)$, which indicates the 4-substitution of imidazolidin-2-one ring (Figure 3).
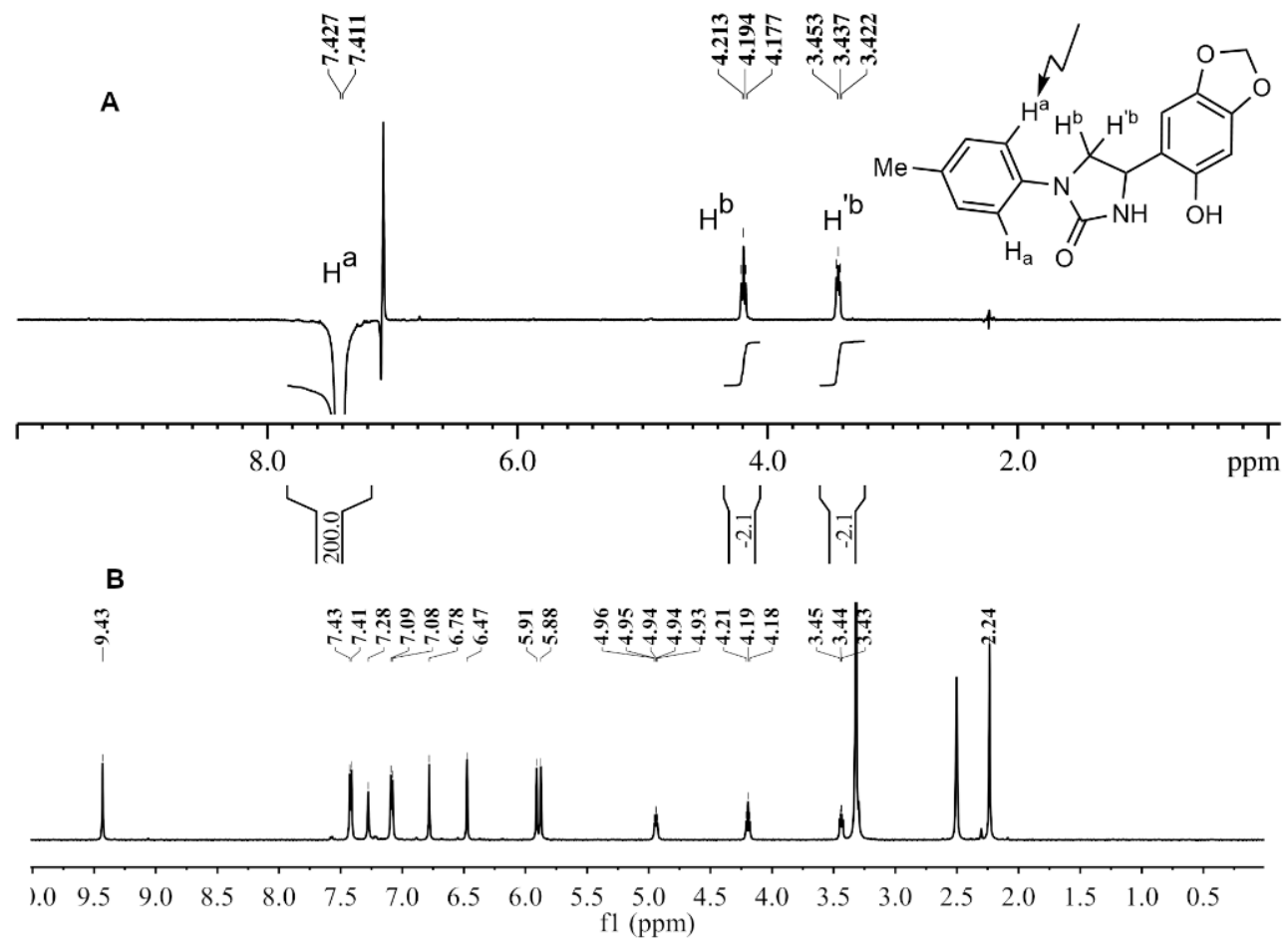

Figure 3. (A) 1D NOESY spectrum of compound 2c (irradiation of ortho-aromatic protons resonance) $\left(500 \mathrm{MHz},\left(\mathrm{CD}_{3}\right)_{2} \mathrm{SO}, 303 \mathrm{~K}\right)$; (B) ${ }^{1} \mathrm{H}$ NMR spectrum of compound $2 \mathrm{c}\left(400 \mathrm{MHz},\left(\mathrm{CD}_{3}\right)_{2} \mathrm{SO}, 303 \mathrm{~K}\right)$. 
Interestingly, the reaction of the urea 1d with 4-hydroxy-6-methylpyran-2-one resulted in the mixture of regioisomeric 4-(2-oxopyran-3-yl)- and 4-(2-oxopyran-5-yl)imidazolidinones $\mathbf{5} \mathbf{d}$ and $\mathbf{5 \mathbf { d } ^ { \prime }}$ ( 10:1 molar ratio according to NMR data). The structure of regioisomers was unequivocally determined by ${ }^{1} \mathrm{H}-{ }^{13} \mathrm{C} \mathrm{HMBC}$ experiments (Figure 4). Thus, in compound $\mathbf{5 d}$, a cross-peak is observed between the protons of the methyl group of the pyran-2-one fragment and $5^{\prime}-\mathrm{C}$ carbon atom, which is bonded to hydrogen. At the same time, in the compound $\mathbf{5} \mathbf{d}^{\prime}$, the cross-peak between the protons of the same methyl group and the substituted $5^{\prime}-\mathrm{C}$ carbon atom is present (see Supplementary Materials, Figure S98, for the $\mathrm{HMBC}$ spectrum). Additionally, there are cross-peaks between the $5^{\prime}-\mathrm{C}$ carbon atom and protons of the methyne $(4-\mathrm{CH})$ and methylene $\left(5-\mathrm{CH}_{2}\right)$ groups of the imidazolidin-2-one ring.

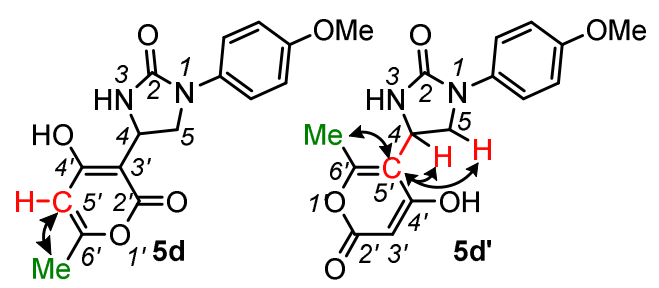

Figure 4. Structure determination of the compounds $\mathbf{5 d}$ and $\mathbf{5 d}^{\prime}$. Key cross-peaks in the ${ }^{1} \mathrm{H}_{-}{ }^{13} \mathrm{C}$ HMBC spectra are indicated by arrows.

The reaction of urea 1a with 2-methylesorcinol possessing two reactive sites resulted in the disubstituted imidazolinone 8 . The compound 8 was obtained as the mixture of the $(R, S)$ - and $(S, S)-,(R, R)$-diastereomers $(d r=1: 1$ according to NMR data), from which one diastereomer was isolated in the individual form in $16 \%$ yield. Unfortunately, we were not able to obtain the crystals suitable for the X-ray analysis; thus, the absolute configuration of the isolated compound could not be determined.

\subsection{Mechanism Discussion and Quantum Chemistry Calculations}

The proposed mechanism of the formation of imidazolidine-2-ones from ureas $\mathbf{1}$ is depicted in Scheme 4 using phenol as the model nucleophile. The first stage of the reaction is the formation of the oxonium cation and its subsequent intramolecular cyclization to give 5-methoxyimidazolidine-2-one $\mathbf{A}$. The acid-promoted elimination of the methanol molecule furnishes iminium cation B. This intermediate may further undergo two concurrent reactions. The first one is the interaction with phenol molecule, which results in the intermediate F via transition state TS2. Further deprotonation of this intermediate results in the 5-substituted imidazolidine-2-one. Alternatively, iminium cation B may undergo deprotonation-protonation steps to give isomeric cation $\mathbf{D}$ via imidazoline-2-one C. The subsequent reaction of cation $\mathbf{D}$ with phenol results in the intermediate $\mathbf{G}$ via transition state TS3. Finally, deprotonation of this intermediate furnishes 4-substituted imidazolidine-2-ones 2.

Quantum chemistry calculations were performed for iminium ions B and D, as well as for $\mathrm{O}$-protonated ion $\mathbf{E}$ to justify the regioselectivity of the reaction. The relative energies of the intermediates are given in Scheme 4. The oxonium ion $\mathbf{E}$ appeared to be the most stable, which is in accordance with the preferred protonation of the ureas at the oxygen atom [21]. The energy of the iminium ion $\mathbf{D}$ is $7.3 \mathrm{kcal} / \mathrm{mol}$ higher compared to cation E. Surprisingly, the isomeric iminium ion $\mathbf{B}$ appeared to be somewhat lower in energy $(6.8 \mathrm{kcal} / \mathrm{mol})$.

Next, energies of the intermediates $\mathbf{F}$ and $\mathbf{G}$, as well as energies of the appropriate transition states TS2 and TS3, were calculated. ortho-Substitution of the phenol was modelled to reflect the presence of the hydroxy group next to the reactive center in the most of the used nucleophiles. Again, the relative energy of the intermediate $\mathbf{F}$ was lower than that of the intermediate G. At the same time, the relative energy of the transition state TS3 appeared to be lower than the energy of transition state TS2 by circa $5 \mathrm{kcal} / \mathrm{mol}$. According to the Curtin-Hammett principle [22], the product distribution for the two rapidly interconverting intermediates depends both on their relative energies and energies of the 
respective transition states. Thus, the observed regioselective formation of 4-substituted imidazolidine-2-ones may be attributed to its lower activation energy.

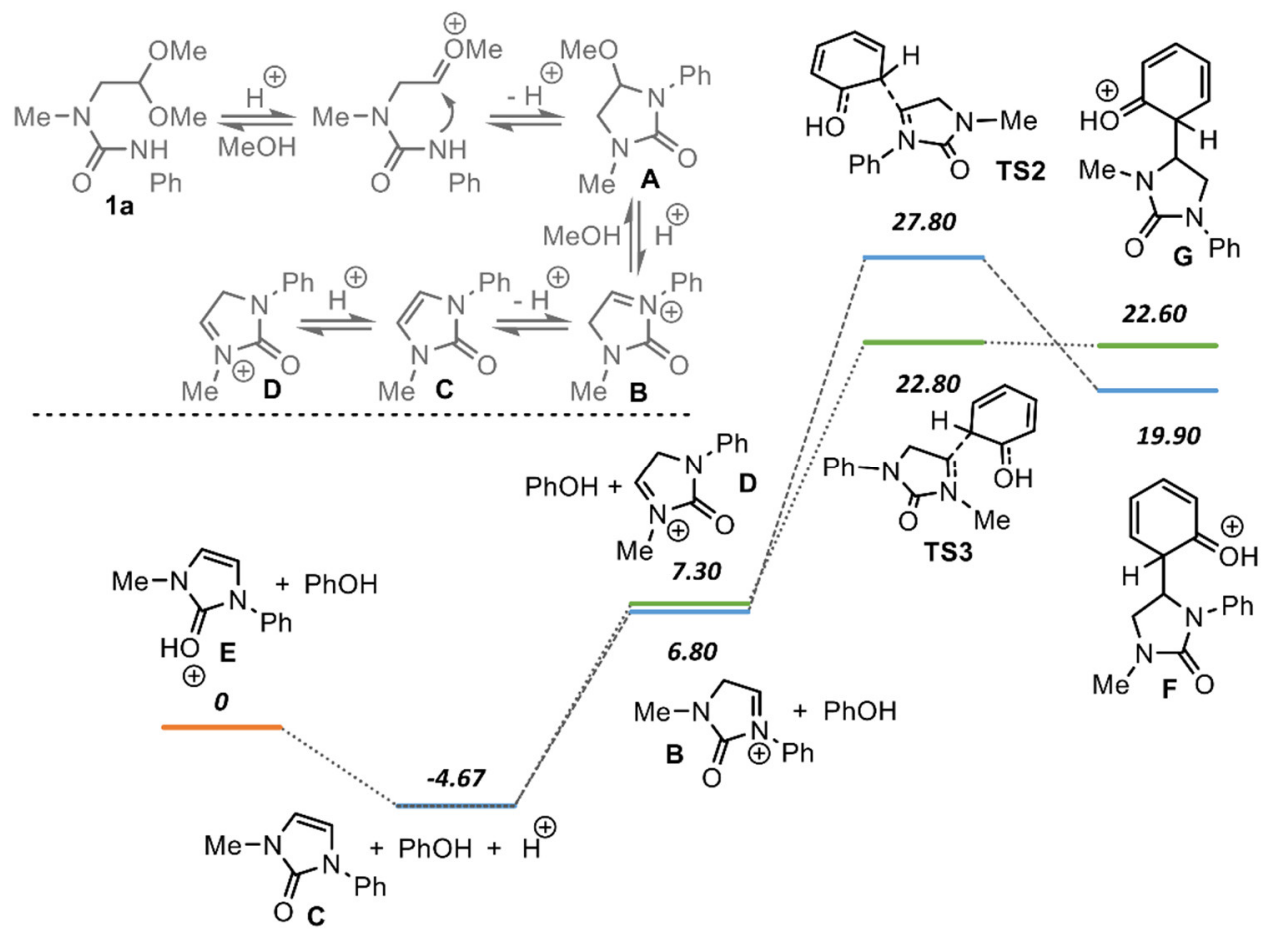

Scheme 4. Plausible mechanism for the formation of regioisomeric imidazolidin-2-ones and relative energies of the intermediates $(\mathrm{kcal} / \mathrm{mol})$, as obtained from quantum chemistry calculations (B3LYP/6$311++G(d, p)$, Gaussian16).

\subsection{Biological Studies}

Some of the resulting compounds were tested for cytotoxicity against normal and cancer human cell lines at concentrations of 1-100 $\mu \mathrm{M}$. As seen from the Table 1, most of the obtained compounds exhibited little to no cytotoxicity against both normal and cancer cells. Compounds $\mathbf{2} \mathbf{h}$ and $\mathbf{2 k}$ appeared to be the most active. The selectivity index (SI) was calculated for them as the ratio between the $\mathrm{IC}_{50}$ value for normal cells and the $\mathrm{IC}_{50}$ value for cancer cells. The cytotoxicity of the compound $2 \mathrm{~h}$ against the HuTu 80 cancer cell line was almost two-fold higher than the cytoxicity against normal cells $(\mathrm{SI}=1.7)$. This is better than the selectivity of the reference compound Arglabin (SI =1.2). Notably, the replacement of the methyl substituent at the nitrogen atom by hydrogen lowers the cytotoxicity against cancer cells, whereas the cytotoxicity against normal cells tends to remain the same or even increases (compare, e.g., $\mathbf{2} \mathbf{h}$ and $\mathbf{2 b}, \mathbf{2 k}$ and $\mathbf{2} \mathbf{d}$ and $\mathbf{2} \mathbf{j}$ and $\mathbf{2 c}$ ). Thus, it appears to be one of the key factors influencing the activity of the compounds under study. 
Table 1. Cytotoxic effects of imidazodin-2-ones 2 on the cancer and normal human cell lines ${ }^{1}$.

\begin{tabular}{|c|c|c|c|c|c|}
\hline \multirow{3}{*}{ Test Compounds } & \multicolumn{4}{|c|}{ Cancer Cell Lines } & \multirow{3}{*}{$\begin{array}{c}\text { Normal Cell Line } \\
\text { Chang Liver } \\
\mathrm{IC}_{50}(\mu \mathrm{M})\end{array}$} \\
\hline & \multicolumn{2}{|c|}{ M-HeLa } & \multicolumn{2}{|c|}{ HuTu 80} & \\
\hline & $\mathrm{IC}_{50}(\mu \mathrm{M})$ & SI & $\mathrm{IC}_{50}(\mu \mathrm{M})$ & SI & \\
\hline $2 \mathrm{~h}$ & $93.6 \pm 8.2$ & 1 & $56 b \pm 5$ & 1.7 & $98.7 \pm 8.8$ \\
\hline $2 j$ & $>100$ & - & $>100$ & - & $>100$ \\
\hline $2 k$ & $59.5 \pm 4.8$ & 1.1 & $56.8 \pm 4.5$ & 1.1 & $63.2 \pm 5.6$ \\
\hline 21 & $96.3 \pm 8.2$ & - & $>100$ & - & $>100$ \\
\hline $2 a$ & $>100$ & - & $>100$ & - & $95.6 \pm 8.2$ \\
\hline $2 b$ & $>100$ & - & $>100$ & - & $87.7 \pm 7.2$ \\
\hline $2 c$ & $>100$ & - & $>100$ & - & $58.4 \pm 4.7$ \\
\hline $2 d$ & $>100$ & - & $>100$ & - & $>100$ \\
\hline $2 e$ & $>100$ & - & $>100$ & - & $>100$ \\
\hline $4 h$ & $98.7 \pm 8.7$ & - & $>100$ & - & $>100$ \\
\hline $4 j$ & $90.7 \pm 7.8$ & - & $>100$ & - & $>100$ \\
\hline $4 k$ & $94.7 \pm 8.8$ & - & $>100$ & - & $85.8 \pm 6.9$ \\
\hline 41 & $97 \pm 8.6$ & - & $>100$ & - & $>100$ \\
\hline $4 c$ & $>100$ & - & $>100$ & - & $>100$ \\
\hline $4 d$ & $>100$ & - & $>100$ & - & $>100$ \\
\hline $5 b$ & $>100$ & - & $>100$ & - & $>100$ \\
\hline $5 c$ & $>100$ & - & $>100$ & - & $>100$ \\
\hline $5 d$ & $>100$ & - & $>100$ & - & $>100$ \\
\hline $6 j$ & $>100$ & - & $>100$ & - & $>100$ \\
\hline $7 \mathrm{c}$ & $>100$ & - & $>100$ & - & $>100$ \\
\hline Tamoxifen & $28.0 \pm 2.5$ & 1.6 & NA & - & $46.2 \pm 3.5$ \\
\hline Arglabin & $41.6 \pm 3.2$ & 0.6 & $22.7 \pm 1.8$ & 1.2 & $26.3 \pm 2.1$ \\
\hline
\end{tabular}

${ }^{1}$ Three independent experiments were carried out-means no selectivity; NA means no activity.

\section{Materials and Methods}

\subsection{Chemistry}

IR spectra were recorded on a UR-20 spectrometer in a $400-3600 \mathrm{~cm}^{-1}$ range in $\mathrm{KBr}$. ${ }^{1} \mathrm{H}$ NMR spectra were recorded on a Bruker MSL 400 spectrometer $(400 \mathrm{MHz})$ with respect to the signals of residual protons of deuterated solvent $\left(\mathrm{CDCl}_{3}, \mathrm{DMSO}_{-} \mathrm{d}_{6}\right) .{ }^{13} \mathrm{C} \mathrm{NMR}$ spectra were recorded on a Bruker Avance $600(151 \mathrm{MHz})$ spectrometer relative to signals of residual protons of deuterated solvent $\left(\mathrm{CDCl}_{3}, \mathrm{DMSO}-\mathrm{d}_{6}\right)$. Elemental analysis is performed on a Carlo Erba device EA 1108. The melting points are determined in glass capillaries on a Stuart SMP 10 instrument.

The X-ray diffraction data for the crystals of $\mathbf{2} \mathbf{j}$ and $\mathbf{2 k}$ were collected on a Bruker D8 Venture automatic diffractometer using graphite monochromated radiation. The structures were solved by direct methods and refined by full-matrix least-squares using the SHELXL97 [23] program. All the non-hydrogen atoms were refined with anisotropic atomic displacement parameters. All figures were made using the program OLEX2 [24]. Crystallographic data for the structure reported in this paper have been deposited with the Cambridge Crystallographic Data Center (2068964-2068965).

Crystal data for $2 \mathbf{j}: \mathrm{C}_{22} \mathrm{H}_{24} \mathrm{~N}_{4} \mathrm{O}_{2}, \mathrm{M}=376.45$, monoclinic, space group $\mathrm{P} 2_{1} / \mathrm{n}$, $\mathrm{Z}=4, \mathrm{a}=7.3485(4), \mathrm{b}=21.8110(10), \mathrm{c}=12.3569(6) \AA, \beta=104.398(2)^{\circ}, \mathrm{V}=1918.34(17) \AA^{3}$, $\varrho_{\text {calc }}=1.303 \mathrm{~g} / \mathrm{cm}^{3}, \mu=0.086 \mathrm{~mm}^{-1}, 15151$ reflections collected $( \pm \mathrm{h}, \pm \mathrm{k}, \pm \mathrm{l}), 3768$ inde- 
pendent (Rint 0.0387 ) and 3013 observed reflections [I $\geq 2 \backslash \mathrm{s}(\mathrm{I})], 257$ refined parameters, $\mathrm{R}_{1}=0.0457, \mathrm{wR}_{2}=0.1049$, max. residual electron density is $0.267(-0.231) \mathrm{e}^{-3}$.

Crystal data for $2 \mathrm{k}: \mathrm{C}_{22} \mathrm{H}_{24} \mathrm{~N}_{4} \mathrm{O}_{3}, \mathrm{M}=392.45$, monoclinic, space group $\mathrm{P} 2{ }_{1} / \mathrm{c}$, $\mathrm{Z}=4, \mathrm{a}=7.5009(5), \mathrm{b}=21.8592(14), \mathrm{c}=12.5236(9) \AA \AA^{\circ}, \beta=107.237(3)^{\circ}, \mathrm{V}=1961.2(2) \AA^{3}$, $\varrho_{\text {calc }}=1.329 \mathrm{~g} / \mathrm{cm}^{3}, \mu=0.091 \mathrm{~mm}^{-1}, 19745$ reflections collected $( \pm \mathrm{h}, \pm \mathrm{k}, \pm \mathrm{l}), 5225$ independent (Rint 0.0637 ) and 3528 observed reflections [I $\geq 2 \backslash \mathrm{s}(\mathrm{I})], 266$ refined parameters, $\mathrm{R}_{1}=0.0479, \mathrm{wR}_{2}=0.1170$, max. residual electron density is $0.258(-0.249) \mathrm{e}^{-3}$.

General method for the synthesis $\mathrm{N}$-(2,2-dimethoxyethyl)ureas 1

To a solution of 2,2-dimethoxyethan-1-amine or 2,2-dimethoxy- $\mathrm{N}$-methylethan-1amine $(11.0 \mathrm{mmol})$ in benzene $(10 \mathrm{~mL})$, aryl isocyanate $(11.0 \mathrm{mmol})$ was added dropwise under cooling $\left(5-8{ }^{\circ} \mathrm{C}\right)$. The reaction mixture was stirred for $6 \mathrm{~h}$ at room temperature, the solvent was removed in vacuum $(20 \mathrm{~mm} \mathrm{Hg})$. The resulting bright fusible solid was dried in vacuum $(1 \mathrm{~h}, 0.01 \mathrm{~mm} \mathrm{Hg})$ to a constant weight.

1-(2,2-Dimethoxyethyl)-3-phenylurea (1a). Yield 93\%. The spectral characteristics were in agreement with the previously published data [25].

1-(4-Chlorophenyl)-3-(2,2-dimethoxyethyl)urea (1b). Yield 95\%; ${ }^{1} \mathrm{H}-\mathrm{NMR}(400 \mathrm{MHz}$, $\left.\mathrm{CHCl}_{3}, \delta \mathrm{ppm}\right) 3.41\left(\mathrm{~s}, 6 \mathrm{H}, \mathrm{CH}_{3}\right), 3.36-3.45\left(\mathrm{~m}, 2 \mathrm{H}, \mathrm{CH}_{2}\right), 4.40(\mathrm{t}, 1 \mathrm{H}, J=4.7 \mathrm{~Hz}, \mathrm{CH})$, 5.68 (br s, 1H, NH), 7.17-7.25 (m, 4H, Ar-H), 7.48 (br s, 1H, NH); ${ }^{13} \mathrm{C}-\mathrm{NMR}(151 \mathrm{MHz}$, $\mathrm{CHCl}_{3}, \delta$ ppm) 41.87, 54.73, 103.69, 121.24, 128.18, 128.98, 137.52, 156.12 .

1-(2,2-Dimethoxyethyl)-3-(p-tolyl)urea (1c). Yield 93\%. The spectral characteristics were in agreement with the previously published data [26].

1-(2,2-Dimethoxyethyl)-3-(4-methoxyphenyl)urea (1d). Yield 97\%; ${ }^{1} \mathrm{H}-\mathrm{NMR}(400 \mathrm{MHz}$, $\left.\mathrm{CHCl}_{3}, \delta \mathrm{ppm}\right) 3.39\left(\mathrm{~s}, 6 \mathrm{H}, \mathrm{CH}_{3}\right), 3.32-3.44\left(\mathrm{~m}, 2 \mathrm{H}, \mathrm{CH}_{2}\right), 3.77\left(\mathrm{~s}, 3 \mathrm{H}, \mathrm{CH}_{3}\right), 4.40(\mathrm{t}, 1 \mathrm{H}$, $J=5.0 \mathrm{~Hz}, \mathrm{CH}), 5.44(\mathrm{br} \mathrm{s}, 1 \mathrm{H}, \mathrm{NH}), 6.82(\mathrm{~d}, 2 \mathrm{H}, J=9.0 \mathrm{~Hz}, \mathrm{Ar}-\mathrm{H}), 7.08(\mathrm{br} \mathrm{s}, 1 \mathrm{H}, \mathrm{NH}), 7.19$ $(\mathrm{d}, 2 \mathrm{H}, J=9.1 \mathrm{~Hz}, \mathrm{Ar}-\mathrm{H}) ;{ }^{13} \mathrm{C}-\mathrm{NMR}\left(151 \mathrm{MHz}, \mathrm{CHCl}_{3}, \delta \mathrm{ppm}\right) 41.90,54.53,55.51,103.56$, $114.45,123.49,131.47,156.43,156.83$.

1-(2,2-Dimethoxyethyl)-3-( $m$-tolyl)urea (1e). Yield 86\%; ${ }^{1} \mathrm{H}-\mathrm{NMR}\left(400 \mathrm{MHz}, \mathrm{CHCl}_{3}, \delta\right.$ ppm) $2.27\left(\mathrm{~s}, 3 \mathrm{H}, \mathrm{CH}_{3}\right), 3.40\left(\mathrm{~s}, 6 \mathrm{H}, \mathrm{CH}_{3}\right), 3.35-3.45\left(\mathrm{~m}, 2 \mathrm{H}, \mathrm{CH}_{2}\right), 4.42(\mathrm{t}, 1 \mathrm{H}, J=5.2 \mathrm{~Hz}$, $\mathrm{CH}), 5.82(\mathrm{br} \mathrm{s}, 1 \mathrm{H}, \mathrm{NH}), 6.84(\mathrm{~d}, 1 \mathrm{H}, J=7.2 \mathrm{~Hz}, \mathrm{Ar}-\mathrm{H}), 7.07-7.18(\mathrm{~m}, 3 \mathrm{H}, \mathrm{Ar}-\mathrm{H}), 7.51$ (br $\mathrm{s}, 1 \mathrm{H}, \mathrm{NH}) ;{ }^{13} \mathrm{C}-\mathrm{NMR}\left(151 \mathrm{MHz}, \mathrm{CHCl}_{3}, \delta \mathrm{ppm}\right) 21.39,41.84,54.48,103.56,117.37,120.99$, $124.02,128.84,138.83,138.89,156.49$.

1-(3-Chlorophenyl)-3-(2,2-dimethoxyethyl)urea (1f). Yield 82\%; ${ }^{1} \mathrm{H}-\mathrm{NMR}\left(400 \mathrm{MHz}, \mathrm{CHCl}_{3}\right.$, $\delta \mathrm{ppm}) 3.42\left(\mathrm{~s}, 6 \mathrm{H}, \mathrm{CH}_{3}\right), 3.37-3.46\left(\mathrm{~m}, 2 \mathrm{H}, \mathrm{CH}_{2}\right), 4.41(\mathrm{t}, 1 \mathrm{H}, J=4.9 \mathrm{~Hz}, \mathrm{CH}), 5.77(\mathrm{br} \mathrm{s}, 1 \mathrm{H}$, $\mathrm{NH}), 6.96(\mathrm{~d}, 1 \mathrm{H}, J=7.4 \mathrm{~Hz}, \mathrm{Ar}-\mathrm{H}), 7.10-7.19(\mathrm{~m}, 2 \mathrm{H}, \mathrm{Ar}-\mathrm{H}), 7.39(\mathrm{~s}, 1 \mathrm{H}, \mathrm{Ar}-\mathrm{H}), 7.65$ (br s, 1H, $\mathrm{NH}) ;{ }^{13} \mathrm{C}-\mathrm{NMR}\left(151 \mathrm{MHz}, \mathrm{CHCl}_{3}, \delta\right.$ ppm) 41.86, 54.69, 103.66, 117.59, 119.62, 122.84, 129.92, $134.56,140.37,155.98$.

1-(2,2-Dimethoxyethyl)-1-methyl-3-phenylurea (1g). Yield 96\%. The spectral characteristics were in agreement with the previously published data [27].

3-(4-Chlorophenyl)-1-(2,2-dimethoxyethyl)-1-methylurea (1h). Yield 91\%; ${ }^{1} \mathrm{H}-\mathrm{NMR}$ (400 $\left.\mathrm{MHz}, \mathrm{CHCl}_{3}, \delta \mathrm{ppm}\right) 3.03\left(\mathrm{~s}, 3 \mathrm{H}, \mathrm{CH}_{3}\right), 3.43\left(\mathrm{~d}, 2 \mathrm{H}, J=5.0 \mathrm{~Hz}, \mathrm{CH}_{2}\right), 3.51\left(\mathrm{~s}, 6 \mathrm{H}, \mathrm{CH}_{3}\right), 4.48$ $(\mathrm{t}, 1 \mathrm{H}, J=5.0 \mathrm{~Hz}, \mathrm{CH}), 7.20-7.24(\mathrm{~m}, 2 \mathrm{H}, \mathrm{Ar}-\mathrm{H}), 7.25-7.30(\mathrm{~m}, 2 \mathrm{H}, \mathrm{Ar}-\mathrm{H}), 7.69$ (br s, $1 \mathrm{H}$, $\mathrm{NH}) ;{ }^{13} \mathrm{C}-\mathrm{NMR}\left(151 \mathrm{MHz}, \mathrm{CHCl}_{3}, \delta \mathrm{ppm}\right) 35.55,52.29,55.04,103.81,119.95,126.72,128.20$, 137.83, 155.97 .

1-(2,2-Dimethoxyethyl)-1-methyl-3-( $p$-tolyl)urea (1j). Yield 90\%; ${ }^{1} \mathrm{H}-\mathrm{NMR}(400 \mathrm{MHz}$, $\left.\mathrm{CHCl}_{3}, \delta \mathrm{ppm}\right) 2.29\left(\mathrm{~s}, 3 \mathrm{H}, \mathrm{CH}_{3}\right), 3.03\left(\mathrm{~s}, 3 \mathrm{H}, \mathrm{CH}_{3}\right), 3.43\left(\mathrm{~d}, 2 \mathrm{H}, J=5.0 \mathrm{~Hz}, \mathrm{CH}_{2}\right), 3.50(\mathrm{~s}$, $\left.6 \mathrm{H}, \mathrm{CH}_{3}\right), 4.48(\mathrm{t}, 1 \mathrm{H}, J=5.0 \mathrm{~Hz}, \mathrm{CH}), 7.07(\mathrm{~d}, 2 \mathrm{H}, J=8.0 \mathrm{~Hz}, \mathrm{Ar}-\mathrm{H}), 7.22(\mathrm{~d}, 2 \mathrm{H}, J=8.2 \mathrm{~Hz}$, Ar-H), 7.47 (br s, $1 \mathrm{H}, \mathrm{NH}) ;{ }^{13} \mathrm{C}-\mathrm{NMR}\left(151 \mathrm{MHz}, \mathrm{CHCl}_{3}, \delta\right.$ ppm) 20.72, 36.06, 52.75, 55.45, $104.39,119.55,129.30,131.92,137.07,156.76$.

1-(2,2-Dimethoxyethyl)-3-(4-methoxyphenyl)-1-methylurea (1k). Yield 96\%; ${ }^{1} \mathrm{H}-\mathrm{NMR}$ $\left(400 \mathrm{MHz}, \mathrm{CHCl}_{3}, \delta \mathrm{ppm}\right) 3.00\left(\mathrm{~s}, 3 \mathrm{H}, \mathrm{CH}_{3}\right), 3.40\left(\mathrm{~d}, 2 \mathrm{H}, J=5.0 \mathrm{~Hz}, \mathrm{CH}_{2}\right), 3.47\left(\mathrm{~s}, 6 \mathrm{H}, \mathrm{CH}_{3}\right)$, 
$3.75\left(\mathrm{~s}, 3 \mathrm{H}, \mathrm{CH}_{3}\right), 4.46(\mathrm{t}, 1 \mathrm{H}, J=5.0 \mathrm{~Hz}, \mathrm{CH}), 6.80(\mathrm{~d}, 2 \mathrm{H}, J=9.0 \mathrm{~Hz}, \mathrm{Ar}-\mathrm{H}), 7.21(\mathrm{~d}, 2 \mathrm{H}$, $J=8.9 \mathrm{~Hz}, \mathrm{Ar}-\mathrm{H}), 7.36$ (br s, $1 \mathrm{H}, \mathrm{NH}) ;{ }^{13} \mathrm{C}-\mathrm{NMR}\left(151 \mathrm{MHz}, \mathrm{CHCl}_{3}, \delta \mathrm{ppm}\right)$ 36.05, 52.61, $55.38,55.51,104.34,114.08,121.53,132.78,155.42,157.00$.

1-(2,2-Dimethoxyethyl)-1-methyl-3-( $m$-tolyl)urea (11). Yield 79\%; ${ }^{1} \mathrm{H}-\mathrm{NMR}(400 \mathrm{MHz}$, $\mathrm{CHCl}_{3}, \delta$ ppm) $2.32\left(\mathrm{~s}, 3 \mathrm{H}, \mathrm{CH}_{3}\right), 3.03\left(\mathrm{~s}, 3 \mathrm{H}, \mathrm{CH}_{3}\right), 3.43\left(\mathrm{~d}, 2 \mathrm{H}, \mathrm{J}=4.7 \mathrm{~Hz}, \mathrm{CH}_{2}\right), 3.50(\mathrm{~s}, 6 \mathrm{H}$, $\left.\mathrm{CH}_{3}\right), 4.48(\mathrm{t}, 1 \mathrm{H}, J=4.7 \mathrm{~Hz}, \mathrm{CH}), 6.81(\mathrm{~d}, 1 \mathrm{H}, J=7.4 \mathrm{~Hz}, \mathrm{Ar}-\mathrm{H}), 7.02-7.11(\mathrm{~m}, 1 \mathrm{H}, \mathrm{Ar}-\mathrm{H})$, 7.12-7.17 (m, 1H, Ar-H), 7.21 (s, 1H, Ar-H), 7.53 (br s, 1H, NH); ${ }^{13} \mathrm{C}-\mathrm{NMR}\left(151 \mathrm{MHz}, \mathrm{CHCl}_{3}\right.$, $\delta$ ppm) 21.50, 36.05, 52.76, 55.47, 104.37, 116.46, 120.10, 123.30, 128.60, 138.60, 139.58, 156.67.

3-(3-Chlorophenyl)-1-(2,2-dimethoxyethyl)-1-methylurea (1m). Yield 80\%; ${ }^{1} \mathrm{H}-\mathrm{NMR}$ (400 $\mathrm{MHz}, \mathrm{CHCl}_{3}, \delta$ ppm) $3.03\left(\mathrm{~s}, 3 \mathrm{H}, \mathrm{CH}_{3}\right), 3.42\left(\mathrm{~d}, 2 \mathrm{H}, \mathrm{J}=5.0 \mathrm{~Hz}, \mathrm{CH}_{2}\right), 3.31\left(\mathrm{~s}, 6 \mathrm{H}, \mathrm{CH}_{3}\right), 4.48$ $(\mathrm{t}, 1 \mathrm{H}, J=4.9 \mathrm{~Hz}, \mathrm{CH}), 6.93-6.98(\mathrm{~m}, 1 \mathrm{H}, \mathrm{Ar}-\mathrm{H}), 7.14-7.21(\mathrm{~m}, 2 \mathrm{H}, \mathrm{Ar}-\mathrm{H}), 7.43$ (s, 1H, Ar-H), 7.75 (br s, $1 \mathrm{H}, \mathrm{NH}) ;{ }^{13} \mathrm{C}-\mathrm{NMR}\left(151 \mathrm{MHz}, \mathrm{CHCl}_{3}, \delta\right.$ ppm) 36.09, 52.82, 55.59, 104.31, 117.24, $119.28,122.36,129.73,134.42,140.99,156.37$.

\section{General method for the synthesis of imidazolidin-2-ones 2}

To a solution of urea $1(0.40 \mathrm{~g}, 1.66 \mathrm{mmol})$ in toluene $(10 \mathrm{~mL})$, the appropriate $C$ nucleophile $(1.66 \mathrm{mmol})$ and TFA $(0.19 \mathrm{~g}, 1.66 \mathrm{mmol})$ were added and the mixture was refluxed for $64 \mathrm{~h}$. Volatiles were removed under vacuum and the residue was washed with acetone, then recrystallized from absolute ethanol and dried under vacuum (10.1 Torr, r.t., $3 \mathrm{~h})$ to give imidazolidin-2-ones 2.

4-(6-Hydroxybenzo[d][1,3]dioxol-5-yl)-1-phenylimidazolidin-2-one (2a). Beige solid, yield 86\%, m.p. $225-226^{\circ} \mathrm{C}$; IR (v, $\left.\mathrm{cm}^{-1}\right)$ : 1598, 1615, 2929, 3113, 3203; ${ }^{1} \mathrm{H}-\mathrm{NMR}(400 \mathrm{MHz}, \mathrm{DMSO}-$ $d_{6}, \delta$ ppm) $3.48\left(\mathrm{dd}, 1 \mathrm{H}, J=6.3 \mathrm{~Hz}, J=9.1 \mathrm{~Hz}, \mathrm{H}_{\mathrm{a}} 3\right), 4.23(\mathrm{dd}, 1 \mathrm{H}, J=8.9 \mathrm{~Hz}, J=9.1 \mathrm{~Hz}$, $\left.\mathrm{H}_{\mathrm{b}} 3\right), 4.96(\mathrm{dd}, 1 \mathrm{H}, J=3.6 \mathrm{~Hz}, J=8.9 \mathrm{~Hz}, \mathrm{H} 4), 5.90(\mathrm{~m}, 2 \mathrm{H}, \mathrm{H} 9), 6.48$ (s, 1H, H11), 6.79 (s, 1H, H7), 6.97 (t, 1H, J = 7.9 Hz, H16), 7.28 (t, 2H, J = 7.9 Hz, H15), 7.36 (s, 1H, NH5), 7.54 (d, $2 \mathrm{H}, J=7.9 \mathrm{~Hz}, \mathrm{H} 14), 9.44(\mathrm{~s}, 1 \mathrm{H}, \mathrm{OH}) ;{ }^{13} \mathrm{C}-\mathrm{NMR}\left(151 \mathrm{MHz}, \mathrm{DMSO}-d_{6}, \delta \mathrm{ppm}\right) 46.6(\mathrm{C} 4)$, 52.0 (C3), 97.6 (C11), 100.7 (C9), 105.6 (C7), 117.0 (C14), 120.1 (C6), 121.5 (C16), 128.5 (C15), 139.8 (C8), 140.5 (C13), 146.6 (C10), 149.2 (C12), 158.4 (C1); Elemental analysis: calc. for $\mathrm{C}_{16} \mathrm{H}_{14} \mathrm{~N}_{2} \mathrm{O}_{4}$ (298): C, 64.42; H, 4.73; N, 9.39; found C, 64.64; H, 4.89; N, 9.27.

1-(4-Chlorophenyl)-4-(6-hydroxybenzo[d][1,3]dioxol-5-yl)imidazolidin-2-one (2b). Beige solid, yield 89\%, m.p. $252{ }^{\circ} \mathrm{C}$; IR ( $\left.v, \mathrm{~cm}^{-1}\right)$ : 1599, 1617, 2906, 3143, 3255; ${ }^{1} \mathrm{H}-\mathrm{NMR}(400 \mathrm{MHz}$, DMSO- $d_{6}, \delta$ ppm) 3.44-3.51 (m, 1H, Ha 3$), 4.18-4.26\left(\mathrm{~m}, 1 \mathrm{H}, \mathrm{H}_{\mathrm{b}} 3\right), 4.92-4.98(\mathrm{~m}, 1 \mathrm{H}, \mathrm{H} 4)$, 5.89 (m, 2H, H9), 6.48 (s, 1H, H11), 6.78 (s, 1H, H7), 7.32 (d, 2H, J = 9.0 Hz, H15), 7.46 (s, 1H, NH5), 7.58 (d, 2H, $J=9.1 \mathrm{~Hz}, \mathrm{H} 16), 9.46(\mathrm{~s}, 1 \mathrm{H}, \mathrm{OH}) ;{ }^{13} \mathrm{C}-\mathrm{NMR}\left(151 \mathrm{MHz}, \mathrm{DMSO}-d_{6}, \delta\right.$ ppm) 46.56 (C4), 51.87 (C3), 97.61 (C11), 100.67 (C9), 105.64 (C7), 118.47 (C14), 119.87 (C6), 125.17 (C16), 128.26 (C15), 139.46 (C8), 139.76 (C13), 146.62 (C10), 149.19 (C12), 158.17 (C1); Elemental analysis: calc. for $\mathrm{C}_{16} \mathrm{H}_{13} \mathrm{ClN}_{2} \mathrm{O}_{4}$ (332.5): C, 57.76; H, 3.94; $\mathrm{Cl}, 10.65 ; \mathrm{N}, 8.42$; found $\mathrm{C}, 57.91 ; \mathrm{H}, 4.14 ; \mathrm{Cl}, 10.83 ; \mathrm{N}, 8.59$.

4-(6-Hydroxybenzo[ $d][1,3]$ dioxol-5-yl)-1-( $p$-tolyl)imidazolidin-2-one (2c). Beige solid, yield 78\%, m.p. 257-258 ${ }^{\circ} \mathrm{C}$; IR (v, $\left.\mathrm{cm}^{-1}\right)$ : 1597, 1627, 2974, 3154, 3275; ${ }^{1} \mathrm{H}-\mathrm{NMR}(400 \mathrm{MHz}, \mathrm{DMSO}-$ $\left.d_{6}, \delta \mathrm{ppm}\right) 2.24(\mathrm{~s}, 3 \mathrm{H}, \mathrm{H} 17), 3.45\left(\mathrm{dd}, 1 \mathrm{H}, J=6.2 \mathrm{~Hz}, J=9.0 \mathrm{~Hz}, \mathrm{H}_{\mathrm{a}} 3\right), 4.20(\mathrm{dd}, 1 \mathrm{H}, J=8.9$ $\left.\mathrm{Hz}, J=9.0 \mathrm{~Hz}, \mathrm{H}_{\mathrm{b}} 3\right), 4.95(\mathrm{dd}, 1 \mathrm{H}, J=3.7 \mathrm{~Hz}, J=8.9 \mathrm{~Hz}, \mathrm{H} 4), 5.90(\mathrm{~d}, 2 \mathrm{H}, J=17.1 \mathrm{~Hz}, \mathrm{H} 9)$, 6.47 (s, 1H, H11), 6.78 (s, 1H, H7), 7.09 (d, 2H, J = 8.4 Hz, H15), 7.29 (br s, 1H, NH5), 7.43 (d, $2 \mathrm{H}, \mathrm{J}=8.6 \mathrm{~Hz}, \mathrm{H} 14), 9.43$ (s, 1H, OH); ${ }^{13} \mathrm{C}-\mathrm{NMR}\left(151 \mathrm{MHz}, \mathrm{DMSO}-d_{6}, \delta \mathrm{ppm}\right) 20.20(\mathrm{C} 17)$, 46.6 (C4), 52.1 (C3), 97.6 (C11), 100.6 (C9), 105.6 (C7), 117.1 (C15), 120.1 (C6), 128.9 (C14), 130.3 (C16), 138.1 (C13), 139.8 (C8), 146.5 (C10), 149.1 (C12), 158.5 (C1); Elemental analysis: calc. for $\mathrm{C}_{17} \mathrm{H}_{16} \mathrm{~N}_{2} \mathrm{O}_{4}$ (312): C, 65.38; $\mathrm{H}, 5.16 ; \mathrm{N}, 8.97$; found $\mathrm{C}, 65.47 ; \mathrm{H}, 5.26 ; \mathrm{N}, 9.19$.

4-(6-Hydroxybenzo[ $d][1,3]$ dioxol-5-yl)-1-(4-methoxyphenyl)imidazolidin-2-one (2d). Beige solid, yield 72\%, m.p. $227-228{ }^{\circ} \mathrm{C}$; IR $\left(v, \mathrm{~cm}^{-1}\right)$ : 1597, 1618, 3153, 3284; ${ }^{1} \mathrm{H}-\mathrm{NMR}(400 \mathrm{MHz}$, DMSO- $d_{6}, \delta$ ppm) 3.39-3.48 (m, 1H, Ha 3), $3.71(\mathrm{~s}, 3 \mathrm{H}, \mathrm{H} 17), 4.14-4.22\left(\mathrm{~m}, 1 \mathrm{H}, \mathrm{H}_{\mathrm{b}} 3\right), 4.90-4.99$ (m, 1H, H4), 5.90 (d, 2H, J = 19.8 Hz, H9), 6.48 (s, 1H, H11), 6.79 (s, 1H, H7), 6.86 (d, 2H, $J=9.1 \mathrm{~Hz}, \mathrm{H} 15), 7.21$ (br s, 1H, NH5), 7.44 (d, 2H, J = 9.0 Hz, H14), 9.42 (s, 1H, OH18); ${ }^{13} \mathrm{C}-$ 
NMR (151 MHz, DMSO-d $d_{6}, \delta$ ppm) 47.22 (C4), 52.92 (C3), 55.68 (C17), 98.14 (C11), 101.18 (C9), 106.12 (C7), 114.31 (C15), 119.38 (C14), 120.70 (C6), 134.41 (C13), 140.30 (C8), 147.06 (C10), 149.65 (C12), 154.83 (C16), 159.14 (C1); Elemental analysis: calc. for $\mathrm{C}_{17} \mathrm{H}_{16} \mathrm{~N}_{2} \mathrm{O}_{5}$ (328): C, 62.19; H, 4.91; N, 8.53; found C, 62.35; H, 5.09; N, 8.75.

4-(6-Hydroxybenzo[d][1,3]dioxol-5-yl)-1-(m-tolyl)imidazolidin-2-one (2e). Beige solid, yield 53\%, m.p. $213{ }^{\circ} \mathrm{C}$; IR ( $\left.v, \mathrm{~cm}^{-1}\right)$ : 1595, 1613, 3153, 3205; ${ }^{1} \mathrm{H}-\mathrm{NMR}\left(400 \mathrm{MHz}, \mathrm{DMSO}-d_{6}\right.$, $\delta$ ppm) 2.27 (s, 3H, H19), 3.42-3.49 (m, 1H, Ha 3 , 4.18-4.27 (m, 1H, H 3 ), 4.91-4.99 (m, 1H, H4), 5.90 (d, 2H, J = $13.7 \mathrm{~Hz}, \mathrm{H} 9), 6.48$ (s, 1H, H11), 6.78 (s, 1H, H7), 6.80 (br s, 1H, NH5), 7.11-7.21 (m, 1H, H14), 7.26-7.41 (m, 3H, H16,17,18), 9.44 (s, 1H, OH20); ${ }^{13} \mathrm{C}-\mathrm{NMR}$ (151 MHz, DMSO- $d_{6}, \delta$ ppm) 21.75 (C19), 47.10 (C4), 52.61 (C3), 98.13 (C11), 101.15 (C9), 106.04 (C7), 114.79 (C14), 118.16 (C16), 120.68 (C18), 122.76 (C17), 128.81 (C15), 138.15 (C8), 140.28 (C13), 147.07 (C10), 149.63 (C12), 158.91 (C1); Elemental analysis: calc. for $\mathrm{C}_{17} \mathrm{H}_{16} \mathrm{~N}_{2} \mathrm{O}_{4}$ (312): C, 65.38; $\mathrm{H}, 5.16 ; \mathrm{N}, 8.97 ;$ found $\mathrm{C}, 65.24 ; \mathrm{H}, 5.32 ; \mathrm{N}, 8.86$.

1-(3-Chlorophenyl)-4-(6-hydroxybenzo[d][1,3]dioxol-5-yl)imidazolidin-2-one (2f). Beige solid, yield 48\%, m.p. 201-202 ${ }^{\circ} \mathrm{C}$; IR ( $\left.v, \mathrm{~cm}^{-1}\right)$ : 1597, 1615, 2943, 3183, 3287; ${ }^{1} \mathrm{H}-\mathrm{NMR}$ (400 MHz, DMSO-d 6 , $\delta$ ppm) 3.46-3.56 (m, 1H, Ha 3), 4.18-4.29 (m, 1H, H 3 ), 4.92-5.02 (m, 1H, H4), 5.90 (d, 2H, J = 15.8 Hz, H9), 6.48 (s, 1H, Ar-H), 6.78 (s, 1H, Ar-H), 6.97-7.04 (m, 1H, Ar-H), 7.26-7.31 (m, 1H, Ar-H), 7.33-7.39 (m, 1H, H11), 7.54 (s, 1H, H7), 7.81 (br s, 1H, NH5), 9.47 (s, 1H, OH19); ${ }^{13} \mathrm{C}-\mathrm{NMR}$ (151 MHz, DMSO-d,$\delta$ ppm) 46.58 (C4), 51.79 (C3), 97.62 (C11), 100.67 (C9), 105.70 (C7), 115.06 (C16), 116.58 (C17), 119.79 (C18), 121.01 (C6), 130.08 (C14), 133.08 (C15), 139.77 (C8), 141.94 (C13), 146.66 (C10), 149.23 (C12), 158.09 (C1); Elemental analysis: calc. for $\mathrm{C}_{16} \mathrm{H}_{13} \mathrm{ClN}_{2} \mathrm{O}_{4}$ (332.5): C, 57.76; H, 3.94; $\mathrm{Cl}, 10.65 ; \mathrm{N}, 8.42$; found $\mathrm{C}, 57.88 ; \mathrm{H}, 4.19 ; \mathrm{Cl}, 10.44 ; \mathrm{N}, 8.42$.

1-Phenyl-4-(6-hydroxybenzo[ $d][1,3]$ dioxol-5-yl)-3-methylimidazolidin-2-one (2g). Yield 91\%. The spectral characteristics were in agreement with the previously published data [19].

1-(4-Chlorophenyl)-4-(6-hydroxybenzo[d][1,3]dioxol-5-yl)-3-methylimidazolidin-2-one (2h). Beige solid, yield 24\%, m.p. $218-219^{\circ} \mathrm{C}$; IR (, $\left.\mathrm{cm}^{-1}\right): 1596,1617,2976,3181,3298 ;{ }^{1} \mathrm{H}-\mathrm{NMR}$ (400 MHz, DMSO- $d_{6}, \delta$ ppm) 2.60 (s, 3H, H5), 3.46-3.55 (m, 1H, Ha 3), 4.08-4.17 (m, 1H, $\mathrm{H}_{\mathrm{b}}$ ) , 4.86-4.94 (m, 1H, H4), $5.92(\mathrm{~d}, 2 \mathrm{H}, J=11.1 \mathrm{~Hz}, \mathrm{H} 9), 6.49$ (s, 1H, H11), 6.68 (s, 1H, H7), $7.33(\mathrm{~d}, 2 \mathrm{H}, J=9.0 \mathrm{~Hz}, \mathrm{H} 15), 7.61(\mathrm{~d}, 2 \mathrm{H}, J=9.1 \mathrm{~Hz}, \mathrm{H} 14), 9.50$ (s, 1H, OH17); ${ }^{13} \mathrm{C}-\mathrm{NMR}$ (151 MHz, DMSO-d 6 , $\delta$ ppm) 28.93 (C5), 49.43 (C4), 52.40 (C3), 97.82 (C11), 100.81 (C9), 106.34 (C7), 116.52 (C14), 118.29 (C15), 125.18 (C16), 128.32 (C14), 139.57 (C8), 140.17 (C13), 147.15 (C10), 150.33 (C12), 157.06 (C1); Elemental analysis: calc. for $\mathrm{C}_{17} \mathrm{H}_{15} \mathrm{ClN}_{2} \mathrm{O}_{4}$ (346.5): C, 58.88; H, 4.36; Cl, 10.22; N, 8.08; found C, 59.00; H, 4.45; Cl, 10.34; N, 8.16.

4-(6-Hydroxybenzo[d][1,3]dioxol-5-yl)-3-methyl-1-( $p$-tolyl)imidazolidin-2-one (2j). Beige solid, yield 32\%, m.p. $182-183{ }^{\circ} \mathrm{C}$; IR $\left(v, \mathrm{~cm}^{-1}\right)$ : 1597, 1618, 2904, 3101, 3189; ${ }^{1} \mathrm{H}-\mathrm{NMR}$ (400 MHz, DMSO-d $d_{6}, \delta$ ppm) 2.24 (s, 3H, H17), 2.59 (s, 3H, H5), 3.42-3.48 (m, 1H, Ha 3 ), 4.05-4.14 (m, 1H, H 3 ), 4.83-4.91 (m, 1H, H4), 5.91 (d, 2H, J = $14.5 \mathrm{~Hz}, \mathrm{H} 9), 6.49(\mathrm{~s}, 1 \mathrm{H}$, H11), 6.67 (s, 1H, H7), 7.10 (d, 2H, J = 8.1 Hz, H15), 7.46 (d, 2H, J = 8.5 Hz, H14), 9.45 (s, 1H, OH18); ${ }^{13}$ C-NMR (151 MHz, DMSO- $d_{6}, \delta$ ppm) 20.75 (C17), 29.62 (C5), 50.20 (C4), 53.01 (C3), 98.37 (C11), 101.33 (C9), 106.64 (C7), 117.35 (C6), 117.54 (C15), 129.48 (C14), 130.94 (C16), 138.73 (C13), 140.73 (C8), 147.59 (C10), 150.79 (C12), 157.96 (C1); Elemental analysis: calc. for $\mathrm{C}_{18} \mathrm{H}_{18} \mathrm{~N}_{2} \mathrm{O}_{4}$ (326): C, 66.25; $\mathrm{H}, 5.56 ; \mathrm{N}, 8.58$; found $\mathrm{C}, 66.39 ; \mathrm{H}, 5.67 ; \mathrm{N}, 8.47$.

4-(6-Hydroxybenzo[d][1,3]dioxol-5-yl)-1-(4-methoxyphenyl)-3-methylimidazolidin-2-one (2k). Beige solid, yield 49\%, m.p. $186-187^{\circ} \mathrm{C}$; IR $\left(v, \mathrm{~cm}^{-1}\right)$ : 1597, 1617, 2978, 3104, 3257; ${ }^{1} \mathrm{H}-\mathrm{NMR}\left(400 \mathrm{MHz}, \mathrm{DMSO}-d_{6}, \delta \mathrm{ppm}\right) 2.59$ (s, 3H, H5), 3.43-3.48 (m, 1H, Ha 3$), 3.71$ (s, 3H, H17), 4.05-4.12 (m, 1H, H 3 ), 4.80-4.90 (m, 1H, H4), 5.91 (d, 2H, J = 14.7 Hz, H9), 6.49 (s, 1H, H11), 6.68 (s, 1H, H7), 6.88 (d, 2H, J = 9.0 Hz, H15), 7.47 (d, 2H, J= 8.9 Hz, H14), 9.46 (s, 1H, OH18); ${ }^{13}$ C-NMR (151 MHz, DMSO-d 6 , $\delta$ ppm) 29.69 (C5), 50.53 (C4), 53.07 (C3), 55.69 (C17), 98.37 (C11), 101.32 (C9), 106.62 (C7), 114.37 (C15), 117.35 (C6), 119.31 (C14), 134.52 (C16), 140.73 (C13), 147.57 (C8), 150.79 (C10), 154.90 (C12), 158.16 (C1); Elemental analysis: calc. for $\mathrm{C}_{18} \mathrm{H}_{18} \mathrm{~N}_{2} \mathrm{O}_{5}$ (342): C, 63.15; $\mathrm{H}, 5.30 ; \mathrm{N}, 8.18$; found $\mathrm{C}, 63.37 ; \mathrm{H}, 5.41 ; \mathrm{N}, 8.21$. 
4-(6-Hydroxybenzo[d][1,3]dioxol-5-yl)-3-methyl-1-( $m$-tolyl)imidazolidin-2-one (21). Beige solid, yield 50\%, m.p. $180{ }^{\circ} \mathrm{C}$; IR $\left(v, \mathrm{~cm}^{-1}\right)$ : 1595, 1615, 2893, 3101, 3179; ${ }^{1} \mathrm{H}-\mathrm{NMR}(400 \mathrm{MHz}$, DMSO- $d_{6}, \delta$ ppm) 2.27 (s, 3H, H19), 2.60 (s, 3H, H5), 3.46-3.54 (m, 1H, Ha 3), 4.07-4.15 (m, $\left.1 \mathrm{H}, \mathrm{H}_{\mathrm{b}} 3\right), 4.85-4.91(\mathrm{~m}, 1 \mathrm{H}, \mathrm{H} 4), 5.91$ (d, 2H, J = $\left.11.5 \mathrm{~Hz}, \mathrm{H} 9\right), 6.50$ (s, 1H, H11), 6.66 (s, 1H, H7), 6.76-6.82 (m, 1H, Ar-H), 7.13-7.20 (m, 1H, Ar-H), 7.36-7.43 (m, 2H, Ar-H), 9.47 (s, 1H, OH20); ${ }^{13}$ C-NMR (151 MHz, DMSO-d 6 , $\delta$ ppm) 21.79 (C19), 29.56 (C5), 50.20 (C3), 52.99 (C4), 98.38 (C11), 101.33 (C9), 106.64 (C7), 114.71 (C14), 117.34 (C6), 118.05 (C18), 122.83 (C16), 128.88 (C17), 138.23 (C15), 140.72 (C13), 141.11 (C8), 147.61 (C10), 150.80 (C12), 157.87 (C1); Elemental analysis: calc. for $\mathrm{C}_{18} \mathrm{H}_{18} \mathrm{~N}_{2} \mathrm{O}_{4}$ (326): $\mathrm{C}, 66.25 ; \mathrm{H}, 5.56 ; \mathrm{N}, 8.58$; found $\mathrm{C}$, 66.16; H, 5.70; N, 8.68.

1-(3-Chlorophenyl)-4-(6-hydroxybenzo[ $d][1,3]$ dioxol-5-yl)-3-methylimidazolidin-2-one (2m). Beige solid, yield 77\%, m.p. $210{ }^{\circ} \mathrm{C}$; IR $\left(v, \mathrm{~cm}^{-1}\right)$ : 1596, 1617, 3134, 3233; ${ }^{1} \mathrm{H}-\mathrm{NMR}(400 \mathrm{MHz}$, DMSO- $d_{6}, \delta$ ppm) 2.60 (s, 3H, H5), 3.49-3.57 (m, 1H, Ha 3$), 4.09-4.18\left(\mathrm{~m}, 1 \mathrm{H}, \mathrm{H}_{\mathrm{b}} 3\right), 4.86-4.94$ (m, 1H, H4), 5.92 (d, 2H, J = 9.4 Hz, H9), 6.49 (s, 1H, H11), 6.70 (s, 1H, H7), 6.98-7.05 (m, 1H, Ar-H), 7.27-7.37 (m, 1H, Ar-H), 7.38-7.46 (m, 1H, Ar-H), 7.79-7.86 (m, 1H, Ar-H), 9.49 (s, 1H, OH19); ${ }^{13} \mathrm{C}-\mathrm{NMR}\left(151 \mathrm{MHz}, \mathrm{DMSO}-d_{6}, \delta \mathrm{ppm}\right) 28.83$ (C5), 49.35 (C3), 52.43 (C4), 97.82 (C11), 100.81 (C9), 106.45 (C7), 114.93 (C18), 116.37 (C17), 116.42 (C16), 121.02 (C6), 130.11 (C14), 133.14 (C15), 140.16 (C13), 142.04 (C8), 147.18 (C10), 150.37 (C12), 156.92 (C1); Elemental analysis: calc. for $\mathrm{C}_{17} \mathrm{H}_{15} \mathrm{ClN}_{2} \mathrm{O}_{4}$ (346.5): C, 59.88; $\mathrm{H}, 4.36 ; \mathrm{Cl}, 10.22 ; \mathrm{N}, 8.08$; found $\mathrm{C}, 58.99 ; \mathrm{H}, 4.49 ; \mathrm{Cl}, 10.46 ; \mathrm{N}, 8.20$.

4-(5-Chloro-2,4-dihydroxyphenyl)-1-phenylimidazolidin-2-one (3a). Beige solid, yield 49\%, m.p. $213-214{ }^{\circ} \mathrm{C}$; IR $\left(v, \mathrm{~cm}^{-1}\right)$ : 1597, 1625, 2902, 3182, 3248, 3348; ${ }^{1} \mathrm{H}-\mathrm{NMR}(400 \mathrm{MHz}$, DMSO- $d_{6}, \delta$ ppm) 3.46-3.54 (m, 1H, Ha 3$), 4.18-4.26\left(\mathrm{~m}, 1 \mathrm{H}, \mathrm{H}_{\mathrm{b}} 3\right), 4.83-4.92(\mathrm{~m}, 1 \mathrm{H}, \mathrm{H} 4)$, 6.56 (s, 1H, H10), 6.97 (t, 1H, J = 7.3 Hz, H17), 7.11 (s, 1H, H7), 7.28 (t, 2H, J = 7.4 Hz, H16), 7.37 (br s, 1H, NH5), 7.54 (d, 2H, J = 7.8 Hz, H15), 9.80 (s, 1H, OH12), 9.94 (s, 1H, OH13); ${ }^{13} \mathrm{C}-\mathrm{NMR}$ (151 MHz, DMSO-d $\left.d_{6}, \delta \mathrm{ppm}\right) 46.32$ (C4), 51.78 (C3), 103.70 (C10), 109.32 (C8), 117.00 (C15), 120.62 (C6), 121.48 (C7), 126.60 (C17), 128.47 (C16), 140.48 (C4), 152.71 (C11), 154.02 (C9), 158.29 (C1); Elemental analysis: calc. for $\mathrm{C}_{15} \mathrm{H}_{13} \mathrm{ClN}_{2} \mathrm{O}_{3}$ (304.5): C, 59.12; $\mathrm{H}$, $4.30 ; \mathrm{Cl}, 11.63 ; \mathrm{N}, 9.19$; found $\mathrm{C}, 59.37 ; \mathrm{H}, 4.54 ; \mathrm{Cl}, 11.81 ; \mathrm{N}, 9.37$.

4-(5-Chloro-2,4-dihydroxyphenyl)-1-(4-methoxyphenyl)imidazolidin-2-one (3d). Beige solid, yield 36\%, m.p. 225-226 ${ }^{\circ} \mathrm{C}$; IR $\left(v, \mathrm{~cm}^{-1}\right): 1594,1626,3182,3249,3345 ;{ }^{1} \mathrm{H}-\mathrm{NMR}$ (400 MHz, DMSO- $d_{6}, \delta$ ppm) 3.42-3.50 (m, 1H, Ha 3$), 3.47$ (s, 3H, H18), 4.15-4.23 (m, 1H, $\left.\mathrm{H}_{\mathrm{b}} 3\right), 4.83-4.91$ (m, 1H, H4), 6.56 (s, 1H, H10), 6.86 (d, 2H, J = 9.2 Hz, H16), 7.12 (s, 1H, H7), 7.24 (br s, 1H, NH5), 7.44 (d, 2H, J = 9.1 Hz, H15), 9.81 (s, 1H, OH12), 9.95 (s, 1H, OH13); ${ }^{13} \mathrm{C}-\mathrm{NMR}\left(151 \mathrm{MHz}, \mathrm{DMSO}-d_{6}, \delta \mathrm{ppm}\right) 46.40$ (C4), 52.21 (C3), 55.12 (C18), 103.69 (C10), 109.34 (C8), 113.77 (C16), 118.82 (C15), 120.70 (C6), 126.58 (C7), 133.83 (C14), 152.67 (C11), 154.00 (C17), 154.29 (C9), 158.52 (C1); Elemental analysis: calc. for $\mathrm{C}_{16} \mathrm{H}_{15} \mathrm{ClN}_{2} \mathrm{O}_{4}$ (334.5): C, 57.41; H, 4.52; Cl, 10.59; N, 8.37; found C, 57.50; H, 4.61; Cl, 10.78; N, 8.36.

4-(5-Chloro-2,4-dihydroxyphenyl)-1-( $m$-tolyl)imidazolidin-2-one (3e). Beige solid, yield 51\%, m.p. 201-202 ${ }^{\circ} \mathrm{C}$; IR $\left(v, \mathrm{~cm}^{-1}\right)$ : 1591, 1628, 2932, 3141, 3209, 3323; ${ }^{1} \mathrm{H}-\mathrm{NMR}(400 \mathrm{MHz}$, DMSO- $d_{6}, \delta$ ppm) $3.27(\mathrm{~s}, 3 \mathrm{H}, \mathrm{H} 20), 3.42-3.52\left(\mathrm{~m}, 1 \mathrm{H}, \mathrm{H}_{\mathrm{a}} 3\right), 4.15-4.24\left(\mathrm{~m}, 1 \mathrm{H}, \mathrm{H}_{\mathrm{a}} 3\right), 4.81-$ 4.92 (m, 1H, H4), 6.56 (s, 1H, H10), 6.76-6.81 (m, 1H, Ar-H), 7.10 (s, 1H, H7), 7.25-7.53 (m, $4 \mathrm{H}, \mathrm{Ar}-\mathrm{H}, \mathrm{NH}), 9.80$ (s, 1H, OH12), 9.94 (s, 1H, OH13); ${ }^{3} \mathrm{C}-\mathrm{NMR}\left(151 \mathrm{MHz}, \mathrm{DMSO}-d_{6}\right.$,

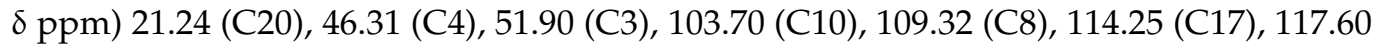
(C19), 120.69 (C6), 122.25 (C15), 126.55 (C7), 128.30 (C18), 137.64 (C16), 140.44 (C14), 152.70 (C11), 154.00 (C9), 158.32 (C1); Elemental analysis: calc. for $\mathrm{C}_{16} \mathrm{H}_{15} \mathrm{ClN}_{2} \mathrm{O}_{3}$ (318.5): C, 60.29; $\mathrm{H}, 4.74 ; \mathrm{Cl}, 11.12 ; \mathrm{N}, 8.79$; found $\mathrm{C}, 60.56 ; \mathrm{H}, 4.80 ; \mathrm{Cl}, 11.26 ; \mathrm{N}, 8.99$.

1,5-Dimethyl-4-(2-oxo-1-phenylimidazolidin-4-yl)-2-phenyl-1,2-dihydro-3H-pyrazol-3-one (4a). Beige solid, yield 44\%, m.p. 199-200 ${ }^{\circ} \mathrm{C}$; IR $\left(v, \mathrm{~cm}^{-1}\right)$ : 1598, 1613, 2969, 3316, 3379; ${ }^{1} \mathrm{H}-\mathrm{NMR}\left(400 \mathrm{MHz}, \mathrm{DMSO}-d_{6}, \delta \mathrm{ppm}\right) 2.29$ (s, 3H, H9), 3.08 (s, 3H, H10), 3.95-4.10 (m, 2H, H3), 4.70-4.79 (m, 1H, H4), 6.97 (t, 1H, J = 7.3 Hz, H14), 7.13 (br s, 1H, NH5), 7.27-7.35 (m, $5 \mathrm{H}, \mathrm{Ar}-\mathrm{H}), 7.48(\mathrm{t}, 2 \mathrm{H}, J=7.8 \mathrm{~Hz}, \mathrm{Ar}-\mathrm{H}), 7.58(\mathrm{~d}, 2 \mathrm{H}, \mathrm{J}=8.4 \mathrm{~Hz}, \mathrm{Ar}-\mathrm{H}) ;{ }^{13} \mathrm{C}-\mathrm{NMR}(151 \mathrm{MHz}$, 
DMSO- $\left.d_{6}, \delta \mathrm{ppm}\right) 10.63$ (C9), 35.36 (C10), 42.61 (C4), 48.74 (C3), 106.39 (C6), 116.85 (C16), 121.29 (C14), 123.50 (C12), 126.12 (C18), 128.45 (C13), 128.94 (C17), 134.99 (C11), 140.61 (C15), 154.84 (C7), 158.10 (C1), 163.78 (C8); Elemental analysis: calc. for $\mathrm{C}_{20} \mathrm{H}_{20} \mathrm{~N}_{4} \mathrm{O}_{2}$ (348): C, 68.95; H, 5.79; N, 16.08; found C, 69.11; H, 5.96; N, 16.22.

4-(1-(4-Chlorophenyl)-2-oxoimidazolidin-4-yl)-1,5-dimethyl-2-phenyl-1,2-dihydro-3Hpyrazol-3-one (4b). Beige solid, yield 64\%, m.p. 203-204 ${ }^{\circ} \mathrm{C}$; IR $\left(v, \mathrm{~cm}^{-1}\right)$ : 1597, 1612, 2954, 3101, 3322; ${ }^{1} \mathrm{H}-\mathrm{NMR}\left(400 \mathrm{MHz}, \mathrm{DMSO}-d_{6}, \delta\right.$ ppm) 2.28 (s, 3H, H9), 3.07 (s, 3H, H10), 3.92-4.00 (m, 1H, H3), 4.02-4.09 (m, 1H, H3), 4.72-4.79 (m, 1H, H4), 7.24 (br s, 1H, NH5), 7.27-7.38 (m, 5H, Ar-H), 7.46-7.51 (m, 2H, Ar-H), 7.61 (d, 2H, J = 9.0 Hz, Ar-H); ${ }^{13} \mathrm{C}-\mathrm{NMR}$ (151 MHz, DMSO-d 6 , $\delta$ ppm) 10.59 (C9), 35.35 (C10), 42.51 (C4), 48.62 (C3), 106.22 (C6), 118.26 (C16), 123.51 (C12), 124.96 (C18), 126.14 (C14), 128.25 (C13), 128.94 (C17), 134.95 (C11), 139.56 (C15), 154.80 (C7), 157.87 (C1), 163.72 (C8); Elemental analysis: calc. for $\mathrm{C}_{20} \mathrm{H}_{19} \mathrm{ClN}_{4} \mathrm{O}_{2}$ (382.5): C, 62.75; H, 5.00; Cl, 9.26; N, 14.63; found C, 62.98; H, 5.21; Cl, 9.29; $\mathrm{N}, 14.49$.

1,5-Dimethyl-4-(2-oxo-1-(p-tolyl)imidazolidin-4-yl)-2-phenyl-1,2-dihydro-3H-pyrazol-3one (4c). Beige solid, yield 47\%, m.p. $213-214{ }^{\circ} \mathrm{C}$; IR $\left(v, \mathrm{~cm}^{-1}\right)$ : 1595, 1615, 3310, 3328; ${ }^{1} \mathrm{H}-\mathrm{NMR}\left(400 \mathrm{MHz}, \mathrm{DMSO}-d_{6}, \delta \mathrm{ppm}\right) 2.24$ (s, 3H, H19), 2.28 (s, 3H, H9), 3.07 (s, 3H, H10), 3.92-3.98 (m, 1H, H3), 3.99-4.05 (m, 1H, H3), 4.69-4.77 (m, 1H, H4), 7.05 (br s, 1H, NH5), $7.10(\mathrm{~d}, 2 \mathrm{H}, \mathrm{J}=8.3 \mathrm{~Hz}, \mathrm{Ar}-\mathrm{H}), 7.28-7.34(\mathrm{~m}, 3 \mathrm{H}, \mathrm{Ar}-\mathrm{H}), 7.44-7.52(\mathrm{~m}, 4 \mathrm{H}, \mathrm{Ar}-\mathrm{H})$; ${ }^{13} \mathrm{C}-\mathrm{NMR}$ (151 MHz, DMSO-d 6 , $\delta$ ppm) 10.64 (C9), 20.21 (C19), 35.37 (C10), 42.66 (C4), 48.85 (C3), 106.44 (C6), 116.94 (C16), 123.49 (C12), 126.11 (C14), 128.86 (C13), 128.94 (C17), 130.11 (C18), 135.00 (C11), 138.18 (C15), 154.84 (C7), 158.18 (C1), 163.79 (C8); Elemental analysis: calc. for $\mathrm{C}_{21} \mathrm{H}_{22} \mathrm{~N}_{4} \mathrm{O}_{2}$ (362): C, 69.59; H, 6.12; N, 15.46; found C, 69.77; H, 6.27; N, 15.55 .

4-(1-(4-Methoxyphenyl)-2-oxoimidazolidin-4-yl)-1,5-dimethyl-2-phenyl-1,2-dihydro-3Hpyrazol-3-one (4d). Beige solid, yield 50\%, m.p. $155^{\circ} \mathrm{C}$; IR $\left(v, \mathrm{~cm}^{-1}\right)$ : 1598, 1613, 2969, 3316, 3379; ${ }^{1} \mathrm{H}-\mathrm{NMR}\left(400 \mathrm{MHz}, \mathrm{DMSO}-d_{6}, \delta \mathrm{ppm}\right) 2.29$ (s, 3H, H9), 3.07 (s, 3H, H10), 3.72 (s, 3H, H19), 3.92-4.07 (m, 2H, H3), 4.68-4.76 (m, 1H, H4), 6.88 (d, 2H, J = 9.0 Hz, Ar-H), 6.99 (br s, 1H, NH5), 7.29-7.35 (m, 3H, Ar-H), 7.46-7.51 (m, 4H, Ar-H); ${ }^{13} \mathrm{C}-\mathrm{NMR}\left(151 \mathrm{MHz}, \mathrm{DMSO}-d_{6}\right.$, $\delta$ ppm) 10.65 (C9), 35.37 (C10), 42.73 (C4), 49.18 (C3), 55.15 (C19), 106.48 (C6), 113.76 (C16), 118.65 (C12), 123.49 (C13), 126.10 (C14), 128.94 (C17), 134.00 (C11), 135.01 (C15), 154.17 (C18), 154.85 (C7), 158.32 (C1), 163.81 (C8); Elemental analysis: calc. for $\mathrm{C}_{21} \mathrm{H}_{22} \mathrm{~N}_{4} \mathrm{O}_{3}(378)$ : C, 66.65; H, 5.86; N, 14.81; found C, 66.41; H, 5.88; N, 14.87.

1,5-Dimethyl-4-(2-oxo-1-( $m$-tolyl)imidazolidin-4-yl)-2-phenyl-1,2-dihydro-3H-pyrazol3-one (4e). Beige solid, yield 57\%, m.p. $185-186{ }^{\circ} \mathrm{C}$; IR $\left(v, \mathrm{~cm}^{-1}\right)$ : 1595, 1617, 2969, 3319; ${ }^{1} \mathrm{H}-\mathrm{NMR}\left(400 \mathrm{MHz}, \mathrm{DMSO}-d_{6}, \delta \mathrm{ppm}\right) 2.28$ (s, 3H,H21), 2.29 (s, 3H, H9), 3.08 (s, 3H, H10), 3.93-4.09 (m, 2H, H3), 4.69-4.77 (m, 1H, H4), 6.79 (d, 1H, J = 7.5 Hz, Ar-H), 7.10 (br s, 1H, NH), 7.17 (t, 1H, J = 7.8 Hz, Ar-H), 7.29-7.34 (m, 3H, Ar-H), 7.36-7.41 (m, 2H, Ar-H), 7.47-7.50 (m, 2H, Ar-H); ${ }^{13} \mathrm{C}-\mathrm{NMR}\left(151 \mathrm{MHz}, \mathrm{DMSO}-d_{6}, \delta \mathrm{ppm}\right) 10.63$ (C9), 21.30 (C21), 35.37 (C10), 42.60 (C4), 48.86 (C3), 106.46 (C6), 114.11 (C16), 117.44 (C20), 122.05 (C18), 123.49 (C12), 126.11 (C19), 128.28 (C14), 128.94 (C13), 135.00 (C17), 137.57 (C11), 140.57 (C15), 154.84 (C7), 158.12 (C1), 163.79 (C8); Elemental analysis: calc. for $\mathrm{C}_{21} \mathrm{H}_{22} \mathrm{~N}_{4} \mathrm{O}_{2}$ (362): C, 69.59; H, 6.12; N, 15.46; found C, 69.72; H, 6.26; N, 15.29.

4-(1-phenyl-3-methyl-2-oxoimidazolidin-4-yl)-1,5-dimethyl-2-phenyl-1,2-dihydro-3Hpyrazol-3-one (4g). Yield 60\%. The spectral characteristics were in agreement with the previously published data [19].

4-(1-(4-Chlorophenyl)-3-methyl-2-oxoimidazolidin-4-yl)-1,5-dimethyl-2-phenyl-1,2-dihydro3H-pyrazol-3-one (4h). Beige solid, yield 43\%, m.p. 245-247 ${ }^{\circ} \mathrm{C}$; IR ( $\left.v, \mathrm{~cm}^{-1}\right)$ : 1595, 1613; ${ }^{1} \mathrm{H}-\mathrm{NMR}\left(400 \mathrm{MHz}, \mathrm{DMSO}-d_{6}, \delta \mathrm{ppm}\right) 2.31$ (s, 3H, H9), 2.62 (s, 3H, H10), 3.13 (s, 3H, H5), 3.91-4.06 (m, 2H, H3), 4.62-4.70 (m, 1H, H4), 7.30-7.37 (m, 5H, Ar-H), 7.46-7.53 (m, 2H, Ar-H), 7.59-7.66 (m, 2H, Ar-H); ${ }^{13} \mathrm{C}-\mathrm{NMR}$ (151 MHz, DMSO- $d_{6}, \delta$ ppm) 10.87 (C9), 28.73 (C5), 35.81 (C10), 46.63 (C3), 48.97 (C4), 102.99 (C6), 118.62 (C16), 124.32 (C12), 125.56 (C18), 126.86 (C14), 128.85 (C13), 129.49 (C17), 135.44 (C11), 140.13 (C15), 156.25 (C7), 157.10 (C1), 
164.26 (C8); Elemental analysis: calc. for $\mathrm{C}_{21} \mathrm{H}_{21} \mathrm{ClN}_{4} \mathrm{O}_{2}$ (396.5): $\mathrm{C}, 63.55 ; \mathrm{H}, 5.33 ; \mathrm{Cl}, 8.93$; $\mathrm{N}, 14.12$; found $\mathrm{C}, 63.69 ; \mathrm{H}, 5.20 ; \mathrm{Cl}, 9.06 ; \mathrm{N}, 14.12$.

1,5-Dimethyl-4-(3-methyl-2-oxo-1-( -tolyl)imidazolidin-4-yl)-2-phenyl-1,2-dihydro-3Hpyrazol-3-one (4j). Beige solid, yield $24 \%$, m.p. $248{ }^{\circ} \mathrm{C}$; IR $\left(v, \mathrm{~cm}^{-1}\right): 1592,1614 ;{ }^{1} \mathrm{H}-$ NMR (400 MHz, DMSO- $d_{6}, \delta$ ppm) 2.24 (s, 3H, H9), 2.30 (s, 3H, H19), 2.60 (s, 3H, H10), 3.12 (s, 3H, H5), 3.90-3.98 (m, 2H, H3), 4.59-4.65 (m, 1H, H4), 7.10 (d, 2H, J = 8.1 Hz, Ar-H), 7.29-7.35 (m, 3H, Ar-H), 7.44-7.51 (m, 4H, Ar-H) ${ }^{13} \mathrm{C}-\mathrm{NMR}$ (151 MHz, DMSO- $d_{6}, \delta$ ppm) 10.93 (C9), 20.75 (C19), 28.91 (C5), 35.86 (C10), 46.77 (C3), 49.22 (C4), 103.21 (C6), 117.34 (C12), 124.30 (C16), 126.84 (C14), 129.48 (C13), 129.51 (C17), 130.78 (C18), 135.50 (C11), 138.80 (C15), 156.31 (C7), 157.49 (C1), 164.38 (C8); Elemental analysis: calc. for $\mathrm{C}_{22} \mathrm{H}_{24} \mathrm{~N}_{4} \mathrm{O}_{2}$ (376): C, 70.19; H, 6.43; N, 14.88; found C, 70.23; H, 6.49; N, 14.88 .

4-(1-(4-Methoxyphenyl)-3-methyl-2-oxoimidazolidin-4-yl)-1,5-dimethyl-2-phenyl-1,2dihydro-3H-pyrazol-3-one (4k). Beige solid, yield 31\%, m.p. $209-210{ }^{\circ} \mathrm{C}$; IR $\left(v, \mathrm{~cm}^{-1}\right)$ : 1595,$1613 ;{ }^{1} \mathrm{H}-\mathrm{NMR}\left(400 \mathrm{MHz}, \mathrm{DMSO}-d_{6}, \delta \mathrm{ppm}\right) 2.31$ (s, 3H, H9), 2.61 (s, 3H, H10), 3.12 (s, 3H, H5), 3.72 (s, 3H, H19), 3.90-3.99 (m, 2H, H3), 4.56-4.65 (m, 1H, H4), 6.90 (d, 2H, $J=9.1 \mathrm{~Hz}, \mathrm{Ar}-\mathrm{H}), 7.32-7.36(\mathrm{~m}, 3 \mathrm{H}, \mathrm{Ar}-\mathrm{H}), 7.42-7.52(\mathrm{~m}, 4 \mathrm{H}, \mathrm{Ar}-\mathrm{H}) ;{ }^{13} \mathrm{C}-\mathrm{NMR}(151 \mathrm{MHz}$, DMSO- $d_{6}, \delta$ ppm) 28.95 (C9), 31.13 (C5), 35.82 (C10), 47.06 (C3), 49.29 (C4), 55.66 (C19), 103.18 (C6), 114.35 (C12), 119.04 (C16), 124.29 (C13), 126.82 (C14), 129.49 (C17), 134.58 (C11), 135.45 (C15), 154.76 (C19), 156.25 (C7), 157.65 (C1), 164.37 (C8); Elemental analysis: calc. for $\mathrm{C}_{22} \mathrm{H}_{24} \mathrm{~N}_{4} \mathrm{O}_{3}$ (392): $\mathrm{C}, 67.33 ; \mathrm{H}, 6.16 ; \mathrm{N}, 14.28$; found $\mathrm{C}, 67.51 ; \mathrm{H}, 6.29 ; \mathrm{N}, 14.40$.

1,5-Dimethyl-4-(3-methyl-2-oxo-1-( $m$-tolyl)imidazolidin-4-yl)-2-phenyl-1,2-dihydro-3Hpyrazol-3-one (41). Beige solid, yield $26 \%$, m.p. $215-216^{\circ} \mathrm{C}$; IR $\left(v, \mathrm{~cm}^{-1}\right): 1592,1613$; ${ }^{1} \mathrm{H}-\mathrm{NMR}\left(400 \mathrm{MHz}\right.$, DMSO- $\left.d_{6}, \delta \mathrm{ppm}\right) 2.28$ (s, 3H, H9), 2.30 (s, 3H, H21), 2.61 (s, 3H, H10), $3.12(\mathrm{~s}, 3 \mathrm{H}, \mathrm{H} 5), 3.91-4.01$ (m, 2H, H3), 4.58-4.67 (m, 1H, H4), $6.80(\mathrm{~d}, 1 \mathrm{H}, J=6.9 \mathrm{~Hz}, \mathrm{Ar}-\mathrm{H})$, $7.17(\mathrm{t}, 1 \mathrm{H}, J=7.5 \mathrm{~Hz}, \mathrm{Ar}-\mathrm{H}), 7.30-7.36(\mathrm{~m}, 3 \mathrm{H}, \mathrm{Ar}-\mathrm{H}), 7.38-7.43(\mathrm{~m}, 2 \mathrm{H}, \mathrm{Ar}-\mathrm{H}), 7.48(\mathrm{t}, 2 \mathrm{H}$, $J=7.4 \mathrm{~Hz}, \mathrm{Ar}-\mathrm{H}) ;{ }^{13} \mathrm{C}-\mathrm{NMR}\left(151 \mathrm{MHz}, \mathrm{DMSO}-d_{6}, \delta \mathrm{ppm}\right) 10.92$ (C9), 21.83 (C21), 28.87 (C5), 35.86 (C10), 46.78 (C3), 49.12 (C4), 103.23 (C6), 114.52 (C16), 117.82 (C18), 122.68 (C20), 124.30 (C12), 126.84 (C14), 128.89 (C19), 129.51 (C13), 135.50 (C17), 138.19 (C11), 141.18 (C15), 156.31 (C7), 157.40 (C1), 164.37 (C8); Elemental analysis: calc. for $\mathrm{C}_{22} \mathrm{H}_{24} \mathrm{~N}_{4} \mathrm{O}_{2}$ (376): C, 70.19; H, 6.43; N, 14.88; found C, 70.37; H, 6.61; N, 14.71 .

4-(4-Hydroxy-6-methyl-2-oxo-2H-pyran-3-yl)-1-phenylimidazolidin-2-one (5a). Beige solid, yield $41 \%$, m.p. $182-183^{\circ} \mathrm{C}$; IR $\left(v, \mathrm{~cm}^{-1}\right)$ : 1598, 1613, 3145, 3271; ${ }^{1} \mathrm{H}-\mathrm{NMR}(400 \mathrm{MHz}$, DMSO$\left.d_{6}, \delta \mathrm{ppm}\right) 2.17(\mathrm{~s}, 3 \mathrm{H}, \mathrm{H} 11), 3.75-3.82\left(\mathrm{~m}, 1 \mathrm{H}, \mathrm{H}_{\mathrm{a}} 3\right), 3.99-4.07\left(\mathrm{~m}, 1 \mathrm{H}, \mathrm{H}_{\mathrm{b}} 3\right), 4.97-5.05(\mathrm{~m}$, $1 \mathrm{H}, \mathrm{H} 4), 6.01(\mathrm{~s}, 1 \mathrm{H}, \mathrm{H} 8), 6.92(\mathrm{br} \mathrm{s}, 1 \mathrm{H}, \mathrm{NH} 5), 6.94(\mathrm{t}, 1 \mathrm{H}, J=7.2 \mathrm{~Hz}, \mathrm{H} 15), 7.27(\mathrm{t}, 2 \mathrm{H}$, $J=7.7 \mathrm{~Hz}, \mathrm{H} 14), 7.57$ (d, 2H, $J=8.4 \mathrm{~Hz}, \mathrm{H} 13), 11.75$ (s, 1H, OH16); ${ }^{13} \mathrm{C}-\mathrm{NMR}(151 \mathrm{MHz}$, DMSO- $d_{6}, \delta$ ppm) 19.87 (C11), 42.57 (C4), 48.84 (C3), 100.41 (C6), 100.79 (C8), 117.04 (C14), 121.38 (C15), 128.92 (C13), 141.44 (C12), 158.81 (C1), 162.54 (C9), 163.44 (C7), 167.55 (C10); Elemental analysis: calc. for $\mathrm{C}_{15} \mathrm{H}_{14} \mathrm{~N}_{2} \mathrm{O}_{4}$ (286): $\mathrm{C}, 62.93 ; \mathrm{H}, 4.93 ; \mathrm{N}, 9.79$; found $\mathrm{C}, 63.12$; H, 5.13; N, 9.91 .

1-(4-Chlorophenyl)-4-(4-hydroxy-6-methyl-2-oxo-2H-pyran-3-yl)imidazolidin-2-one (5b). Beige solid, yield $66 \%$, m.p. $190-191{ }^{\circ} \mathrm{C}$; IR $\left(v, \mathrm{~cm}^{-1}\right): 1592,1613,3102,3228 ;{ }^{1} \mathrm{H}-\mathrm{NMR}$ (400 MHz, DMSO- $d_{6}, \delta$ ppm) $2.18(\mathrm{~s}, 3 \mathrm{H}, \mathrm{H} 11), 3.77-3.80\left(\mathrm{~m}, 1 \mathrm{H}, \mathrm{H}_{\mathrm{a}} 3\right), 4.00-4.10(\mathrm{~m}, 1 \mathrm{H}$, $\left.\mathrm{H}_{\mathrm{b}} 3\right), 4.96-5.05(\mathrm{~m}, 1 \mathrm{H}, \mathrm{H} 4), 6.02(\mathrm{~s}, 1 \mathrm{H}, \mathrm{H} 8), 7.05$ (br s, $\left.1 \mathrm{H}, \mathrm{NH} 5\right), 7.32(\mathrm{~d}, 2 \mathrm{H}, J=9.1 \mathrm{~Hz}$, $\mathrm{H} 14), 7.61(\mathrm{~d}, 2 \mathrm{H}, J=9.0 \mathrm{~Hz}, \mathrm{H} 13), 11.80(\mathrm{~s}, 1 \mathrm{H}, \mathrm{OH} 16) ;{ }^{13} \mathrm{C}-\mathrm{NMR}\left(151 \mathrm{MHz}, \mathrm{DMSO}-d_{6}, \delta\right.$ ppm) 19.84 (C11), 42.46 (C4), 48.80 (C3), 100.38 (C6), 100.69 (C8), 118.45 (C14), 125.03 (C15), 128.70 (C13), 140.36 (C12), 158.59 (C1), 162.57 (C9), 163.39 (C7), 167.55 (C10); Elemental analysis: calc. for $\mathrm{C}_{15} \mathrm{H}_{13} \mathrm{ClN}_{2} \mathrm{O}_{4}$ (320.5): $\mathrm{C}, 56.17 ; \mathrm{H}, 4.09 ; \mathrm{Cl}, 11.05 ; \mathrm{N}, 8.73$; found $\mathrm{C}, 55.98$; $\mathrm{H}, 3.95 ; \mathrm{Cl}, 10.87 ; \mathrm{N}, 8.68$.

4-(4-Hydroxy-6-methyl-2-oxo-2H-pyran-3-yl)-1-( $p$-tolyl)imidazolidin-2-one (5c). Beige solid, yield $86 \%$, m.p. $184-185^{\circ} \mathrm{C}$; IR $\left(v, \mathrm{~cm}^{-1}\right)$ : 1593, 1620, 3132, 3288; ${ }^{1} \mathrm{H}-\mathrm{NMR}(400 \mathrm{MHz}$, DMSO$\left.d_{6}, \delta \mathrm{ppm}\right) 2.17(\mathrm{~s}, 3 \mathrm{H}, \mathrm{H} 11), 2.24(\mathrm{~s}, 3 \mathrm{H}, \mathrm{H} 16), 3.74-3.82\left(\mathrm{~m}, 1 \mathrm{H}, \mathrm{H}_{\mathrm{a}} 3\right), 3.96-4.05(\mathrm{~m}, 1 \mathrm{H}$, $\left.\mathrm{H}_{\mathrm{b}} 3\right), 4.95-5.02(\mathrm{~m}, 1 \mathrm{H}, \mathrm{H} 4), 6.01(\mathrm{~s}, 1 \mathrm{H}, \mathrm{H} 8), 6.84(\mathrm{br} \mathrm{s}, 1 \mathrm{H}, \mathrm{NH} 5), 7.08(\mathrm{~d}, 2 \mathrm{H}, J=8.4 \mathrm{~Hz}$, 
$\mathrm{H} 14), 7.45(\mathrm{~d}, 2 \mathrm{H}, J=8.5 \mathrm{~Hz}, \mathrm{H} 13), 11.74(\mathrm{~s}, 1 \mathrm{H}, \mathrm{OH} 17) ;{ }^{13} \mathrm{C}-\mathrm{NMR}\left(151 \mathrm{MHz}, \mathrm{DMSO}-d_{6}, \delta\right.$ ppm) 19.32 (C11), 20.19 (C16), 42.08 (C4), 48.36 (C3), 99.88 (C6), 100.25 (C8), 116.57 (C14), 128.79 (C13), 129.59 (C15), 138.49 (C12), 158.34 (C1), 161.96 (C9), 162.89 (C7), 167.00 (C10); Elemental analysis: calc. for $\mathrm{C}_{16} \mathrm{H}_{16} \mathrm{~N}_{2} \mathrm{O}_{4}$ (300): C, 63.99; $\mathrm{H}, 5.37 ; \mathrm{N}, 9.33$; found C, 64.09; $\mathrm{H}, 5.47 ; \mathrm{N}, 9.49$.

4-(4-Hydroxy-6-methyl-2-oxo-2H-pyran-3-yl)-1-(4-methoxyphenyl)imidazolidin-2-one (5d). Beige solid, yield 84\%, m.p. $184-185^{\circ} \mathrm{C}$; IR $\left(v, \mathrm{~cm}^{-1}\right)$ : 1593, 1614, 3218; ${ }^{1} \mathrm{H}-\mathrm{NMR}(400 \mathrm{MHz}$, DMSO- $d_{6}, \delta$ ppm) 2.17 (s, 3H, H11), 3.71 (s, 3H, H16), 3.76-3.78 (m, 1H, Ha 3), 3.96-4.02 (m, $\left.1 \mathrm{H}, \mathrm{H}_{\mathrm{b}} 3\right), 4.94-5.00$ (m, 1H, H4), 6.01 (s, 1H, H8), 6.78 (br s, 1H, NH5), 6.86 (d, 2H, J = 9.2 $\mathrm{Hz}, \mathrm{H} 14), 7.47$ (d, 2H, $J=9.1 \mathrm{~Hz}, \mathrm{H} 13), 11.74$ (s, 1H, OH17); ${ }^{13} \mathrm{C}-\mathrm{NMR}\left(151 \mathrm{MHz}, \mathrm{DMSO}-d_{6}\right.$,

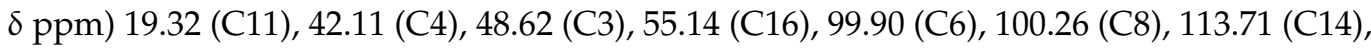
118.22 (C13), 134.37 (C12), 153.84 (C15), 158.44 (C1), 161.93 (C9), 162.90 (C7), 167.01 (C10); Elemental analysis: calc. for $\mathrm{C}_{16} \mathrm{H}_{16} \mathrm{~N}_{2} \mathrm{O}_{5}$ (316): C, 60.76; $\mathrm{H}, 5.10 ; \mathrm{N}, 8.86$; found $\mathrm{C}, 60.87$; H, 5.26; N, 8.99.

1-(3-Chlorophenyl)-4-(4-hydroxy-6-methyl-2-oxo-2H-pyran-3-yl)imidazolidin-2-one (5f). Beige solid, yield 53\%, m.p. $194{ }^{\circ} \mathrm{C}$; IR $\left(v, \mathrm{~cm}^{-1}\right)$ : 1598, 1613, 3125, 3221; ${ }^{1} \mathrm{H}-\mathrm{NMR}(400 \mathrm{MHz}$, DMSO- $d_{6}, \delta$ ppm) $2.17(\mathrm{~s}, 3 \mathrm{H}, \mathrm{H} 11), 3.74-3.80\left(\mathrm{~m}, 1 \mathrm{H}, \mathrm{H}_{\mathrm{a}} 3\right), 4.02-4.10\left(\mathrm{~m}, 1 \mathrm{H}, \mathrm{H}_{\mathrm{b}} 3\right), 4.93-$ 5.04 (m, 1H, H4), 6.01 (s, 1H, H8), 6.95-7.00 (m, 1H, Ar-H), 7.12 (br s, 1H, NH5), 7.27-7.32 (m, 1H, Ar-H), 7.33-7.37 (m, 1H, Ar-H), 7.86 (s, 1H, H13), 11.79 (s, 1H, OH18); ${ }^{13} \mathrm{C}-\mathrm{NMR}$ (151 MHz, DMSO-d $d_{6} \delta$ ppm) 19.34 (H11), 41.92 (H4), 48.27 (H3), 99.86 (H6), 100.18 (H8), 114.55 (H17), 116.00 (H15), 120.37 (H16), 130.05 (H13), 133.06 (H14), 142.27 (H12), 158.00 (H1), 162.08 (H9), 162.89 (H7), 167.05 (H10); Elemental analysis: calc. for $\mathrm{C}_{15} \mathrm{H}_{13} \mathrm{ClN}_{2} \mathrm{O}_{4}$ (320): C, 56.17; H, 4.09; Cl, 11.05; N, 8.73; found C, 56.15; H, 4.14; Cl, 11.05; N, 8.81.

4-(4-Hydroxy-2-oxo-2H-chromen-3-yl)-1-phenylimidazolidin-2-one (6a). Beige solid, yield 91\%, m.p. $193-194{ }^{\circ} \mathrm{C}$; IR ( $\left.v, \mathrm{~cm}^{-1}\right)$ : 1596, 1614, 3131, 3203; ${ }^{1} \mathrm{H}-\mathrm{NMR}$ (400 MHz, DMSO- $d_{6}$,

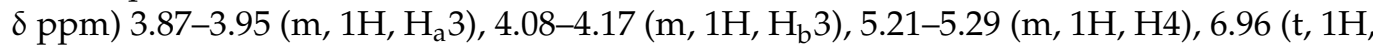
$J=7.3 \mathrm{~Hz}, \mathrm{Ar}-\mathrm{H}), 7.04$ (br s, 1H, NH5), 7.27-7.33 (m, 2H, Ar-H), 7.36-7.42 (m, 2H, Ar-H), $7.61(\mathrm{~d}, 2 \mathrm{H}, J=7.8 \mathrm{~Hz}, \mathrm{Ar}-\mathrm{H}), 7.63-7.67(\mathrm{~m}, 1 \mathrm{H}, \mathrm{Ar}-\mathrm{H}), 8.04(\mathrm{dd}, 1 \mathrm{H}, J=7.8, J=1.6, \mathrm{~Hz}$, Ar-H); ${ }^{13}$ C-NMR (151 MHz, DMSO-d 6 , $\delta$ ppm) 42.77 (C4), 48.47 (C3), 104.51 (C6), 116.10 (C8), 116.29 (C12), 116.53 (C16), 120.97 (C9), 123.51 (C10), 123.92 (C18), 128.43 (C17), 132.51 (C11), 140.80 (C15), 152.32 (C13), 158.34 (C1), 161.03 (C7), 162.09 (C14); Elemental analysis: calc. for $\mathrm{C}_{18} \mathrm{H}_{14} \mathrm{~N}_{2} \mathrm{O}_{4}$ (322): C, 67.08; $\mathrm{H}, 4.38 ; \mathrm{N}, 8.69$; found $\mathrm{C}, 67.19 ; \mathrm{H}, 4.50 ; \mathrm{N}, 8.75$.

1-(4-Chlorophenyl)-4-(4-hydroxy-2-oxo-2H-chromen-3-yl)imidazolidin-2-one (6h). Beige solid, yield 58\%, m.p. $199-200{ }^{\circ} \mathrm{C}$; IR $\left(v, \mathrm{~cm}^{-1}\right)$ : 1597, 1616, 3284; ${ }^{1} \mathrm{H}-\mathrm{NMR}(400 \mathrm{MHz}$, DMSO- $d_{6}, \delta$ ppm) 3.85-3.93 (m, 1H, Ha 3$), 4.08-4.16\left(\mathrm{~m}, 1 \mathrm{H}, \mathrm{H}_{\mathrm{b}} 3\right), 5.23-5.31(\mathrm{~m}, 1 \mathrm{H}, \mathrm{H} 4)$, 7.15 (br s, 1H, NH5), 7.32-7.35 (m, 2H, Ar-H), 7.36-7.42 (m, 2H, Ar-H), 7.62-7.67 (m, 3H, Ar-H), 8.03 (dd, $1 \mathrm{H}, J=8.0, J=1.5, \mathrm{~Hz}, \mathrm{Ar}-\mathrm{H}) ;{ }^{13} \mathrm{C}-\mathrm{NMR}\left(151 \mathrm{MHz}, \mathrm{DMSO}-d_{6}, \delta\right.$ ppm $) 42.67$ (C4), 48.44 (C3), 104.41 (C6), 116.09 (C8), 116.30 (C12), 117.96 (C17), 123.53 (C9), 123.93 (C10), 124.66 (C18), 128.24 (C16), 132.53 (C11), 139.74 (C15), 152.33 (C13), 158.17 (C1), 161.05 (C7), 162.17 (C14); Elemental analysis: calc. for $\mathrm{C}_{18} \mathrm{H}_{13} \mathrm{ClN}_{2} \mathrm{O}_{4}(356.5): \mathrm{C}, 60.60 ; \mathrm{H}, 3.67 ; \mathrm{Cl}$, 9.94; N, 7.85; found C, 60.65; H, 3.64; Cl, 9.91; N, 7.94.

4-(4-Hydroxy-2-oxo-2H-chromen-3-yl)-1-( $p$-tolyl)imidazolidin-2-one (6j). Beige solid, yield $68 \%$, m.p. $194-196{ }^{\circ} \mathrm{C}$; IR $\left(v, \mathrm{~cm}^{-1}\right)$ : 1598, 1612, 3124, 3244; ${ }^{1} \mathrm{H}-\mathrm{NMR}\left(400 \mathrm{MHz}, \mathrm{DMSO}-d_{6}, \delta\right.$ ppm) 2.25 (s, 3H, H19), 3.84-3.94 (m, 1H, Ha 3), 4.03-4.14 (m, 1H, $\left.\mathrm{H}_{\mathrm{b}} 3\right), 5.19-5.29(\mathrm{~m}, 1 \mathrm{H}$, H)4, 6.96 (br s, 1H, NH5), 7.10 (d, 2H, J = 8.1 Hz, Ar-H), 7.33-7.42 (m, 2H, Ar-H), 7.49 (d, $2 \mathrm{H}, J=8.3 \mathrm{~Hz}, \mathrm{Ar}-\mathrm{H}), 7.65(\mathrm{t}, 1 \mathrm{H}, J=7.1 \mathrm{~Hz}, \mathrm{Ar}-\mathrm{H}), 8.00-8.07$ (m, 1H, Ar-H); ${ }^{13} \mathrm{C}-\mathrm{NMR}$ (151 MHz, DMSO- $d_{6}, \delta$ ppm) 20.75 (C19), 43.41 (C4), 49.12 (C3), 105.03 (C6), 116.66 (C8), 116.82 (C12), 117.18 (C17), 124.04 (C9), 124.45 (C10), 129.38 (C16), 130.31 (C18), 133.02 (C11), 138.94 (C15), 152.87 (C13), 158.94 (C1), 161.55 (C7), 162.61 (C14); Elemental analysis: calc. for $\mathrm{C}_{19} \mathrm{H}_{16} \mathrm{~N}_{2} \mathrm{O}_{4}$ (336): C, 67.85; $\mathrm{H}, 4.80 ; \mathrm{N}, 8.33$; found $\mathrm{C}, 68.08 ; \mathrm{H}, 4.96 ; \mathrm{N}, 8.45$.

2-(1-(4-Chlorophenyl)-2-oxoimidazolidin-4-yl)-3-hydroxynaphthalene-1,4-dione (7b). Beige solid, yield 86\%, m.p. $234-235^{\circ} \mathrm{C}$; IR $\left(v, \mathrm{~cm}^{-1}\right)$ : 1598, 1600, 1613, 3154, 3221; ${ }^{1} \mathrm{H}-\mathrm{NMR}$ 
(400 MHz, DMSO-d $d_{6}, \delta$ ppm) 3.82-3.91 (m, 1H, Ha 3$), 4.12-4.21\left(\mathrm{~m}, 1 \mathrm{H}, \mathrm{H}_{\mathrm{b}} 3\right), 5.15-5.24(\mathrm{~m}$, 1H, H4), 7.13 (br s, 1H, NH5), 7.34 (d, 2H, J = 9.0 Hz, H18), 7.63 (d, 2H, J = 9.0 Hz, H17), 7.79-7.88 (m, 2H, Ar-H), 7.98-8.02 (m, 2H, Ar-H); ${ }^{13} \mathrm{C}-\mathrm{NMR}\left(151 \mathrm{MHz}, \mathrm{DMSO}-d_{6}, \delta \mathrm{ppm}\right)$ 41.76 (C4), 48.58 (C3), 117.96 (C18), 120.97 (C6), 124.66 (C13), 125.72 (C9), 125.81 (C12), 128.26 (C17), 129.77 (C8), 132.02 (C19), 133.24 (C11), 134.88 (C10), 139.74 (C16), 156.86 (C15), 158.12 (C1), 181.19 (C14), 183.39 (C7); Elemental analysis: calc. for $\mathrm{C}_{19} \mathrm{H}_{13} \mathrm{ClN}_{2} \mathrm{O}_{4}(368.5)$ : C, 61.88; H, 3.55; Cl, 9.61; N, 7.60; found C, 62.09; H, 3.77; Cl, 9.57; N, 7.47.

2-Hydroxy-3-(2-oxo-1-( $p$-tolyl)imidazolidin-4-yl)naphthalene-1,4-dione (7c). Beige solid, yield $60 \%$, m.p. $223-224{ }^{\circ} \mathrm{C}$; IR $\left(v, \mathrm{~cm}^{-1}\right)$ : 1597, 1600, 1622, 3133, 3287; ${ }^{1} \mathrm{H}-\mathrm{NMR}(400 \mathrm{MHz}$, DMSO-d $d_{6}, \delta$ ppm) 2.25 (s, 3H, H20), 3.82-3.90 (m, 1H, Ha 3), 4.08-4.19 (m, 1H, H 3 ), 5.155.23 (m, 1H, H4), 6.94 (br s, 1H, NH5), 7.10 (d, 2H, J = 8.3 Hz, H18), 7.48 (d, 2H, J = 8.6 Hz, H17), 7.79-7.83 (m, 1H, Ar-H), 7.85-7.90 (m, 1H, Ar-H), 7.97-8.04 (m, 2H, Ar-H); ${ }^{13} \mathrm{C}-\mathrm{NMR}$ (151 MHz, DMSO-d 6 , $\delta$ ppm) 20.22 (C20), 41.89 (C4), 48.71 (C3), 116.63 (C18), 120.51 (C13), 121.18 (C6), 125.70 (C9), 125.81 (C12), 128.85 (C17), 129.78 (C8), 132.02 (C11), 133.23 (C10), 134.88 (C19), 138.39 (C16), 156.74 (C15), 158.37 (C1), 181.21 (C14), 183.44 (C7); Elemental analysis: calc. for $\mathrm{C}_{20} \mathrm{H}_{16} \mathrm{~N}_{2} \mathrm{O}_{4}$ (348): C, 68.96; $\mathrm{H}, 4.63 ; \mathrm{N}, 8.04$; found $\mathrm{C}, 69.15 ; \mathrm{H}, 4.73$; $\mathrm{N}, 8.13$.

1-(3-Chlorophenyl)-4-(4-hydroxy-2-oxo-2H-chromen-3-yl)imidazolidin-2-one (7f). Beige solid, yield 83\%, m.p. $199{ }^{\circ} \mathrm{C}$; IR ( $\left.v, \mathrm{~cm}^{-1}\right)$ : 1594, 1618, 3176, 3293; ${ }^{1} \mathrm{H}-\mathrm{NMR}(400 \mathrm{MHz}$, DMSO- $d_{6}, \delta$ ppm) 3.85-3.95 (m, 1H, Ha 3$), 4.08-4.19\left(\mathrm{~m}, 1 \mathrm{H}, \mathrm{H}_{\mathrm{b}} 3\right), 5.22-5.30(\mathrm{~m}, 1 \mathrm{H}, \mathrm{H} 4)$, 6.98-7.02 (m, 1H, Ar-H), 7.22 (br s, 1H, NH5), 7.29-7.33 (m, 1H, Ar-H), 7.36-7.42 (m, 3H, Ar-H), 7.62-7.67 (m, 1H, Ar-H), 7.89 (s, 1H, Ar-H), 8.01-8.06 (m, 1H, Ar-H); ${ }^{13} \mathrm{C}-\mathrm{NMR}$ (151 MHz, DMSO- $d_{6}, \delta$ ppm) 42.65 (C4), 48.42 (C3), 104.40 (C6), 114.61 (C19), 116.05 (C8), 116.10 (C12), 116.31 (C18), 120.50 (C20), 123.54 (C9), 123.93 (C10), 130.09 (C17), 132.54 (C11), 133.10 (C16), 142.19 (C15), 152.33 (C13), 158.10 (C1), 161.05 (C7), 162.19 (C14); Elemental analysis: calc. for $\mathrm{C}_{18} \mathrm{H}_{13} \mathrm{ClN}_{2} \mathrm{O}_{4}$ (356.5): $\mathrm{C}, 60.60 ; \mathrm{H}, 3.67 ; \mathrm{Cl}, 9.94 ; \mathrm{N}, 7.85$; found $\mathrm{C}, 60.78$; $\mathrm{H}, 3.81 ; \mathrm{Cl}, 10.09 ; \mathrm{N}, 7.86$.

4,4'-(4,6-Dihydroxy-5-methyl-1,3-phenylene)bis(1-(4-chlorophenyl)imidazolidin-2-one) (8). Beige solid, yield 16\%, m.p. > $250{ }^{\circ} \mathrm{C}$; IR $\left(v, \mathrm{~cm}^{-1}\right): 1594,1627,3089,3171,3276$; ${ }^{1} \mathrm{H}-$ NMR (400 MHz, DMSO- $d_{6}, \delta$ ppm) 2.10 (s, 3H, H10), 3.42-3.51 (m, 2H, H 3 3), 4.21-4.30 (m, $\left.2 \mathrm{H}, \mathrm{H}_{\mathrm{b}} 3\right), 5.00-5.08(\mathrm{~m}, 2 \mathrm{H}, \mathrm{H} 4), 7.16(\mathrm{~s}, 1 \mathrm{H}, \mathrm{H} 9), 7.32$ (d, 4H, J = 8.9 Hz, H13), 7.52 (br s, 2H, NH5), 7.59 (d, 4H, $J=8.9 \mathrm{~Hz}, \mathrm{H} 12), 7.50$ (s, 2H, OH15); ${ }^{13} \mathrm{C}-\mathrm{NMR}\left(151 \mathrm{MHz}, \mathrm{DMSO}-d_{6}, \delta\right.$ ppm) 10.20 (C10), 47.62 (C4), 52.76 (C3), 113.55 (C8), 118.87 (C13), 120.73 (C6), 121.45 (C14), 125.61 (C9), 128.82 (C12), 140.09 (C11), 152.68 (C7), 158.73 (C1); Elemental analysis: calc. for $\mathrm{C}_{25} \mathrm{H}_{22} \mathrm{Cl}_{2} \mathrm{~N}_{4} \mathrm{O}_{4}$ (513): C, 58.49; $\mathrm{H}, 4.32 ; \mathrm{Cl}, 13.81 ; \mathrm{N}, 10.91$; found $\mathrm{C}, 58.78 ; \mathrm{H}, 4.58 ; \mathrm{Cl}$, 13.99; N, 11.09 .

\subsection{Biological Studies}

Cytotoxic effects of the test compounds on human cancer and normal cells were estimated by means of the multifunctional Cytell Cell Imaging system (GE Health Care Life Science, Sweden) using the Cell Viability Bio App, which precisely counts the number of cells and evaluates their viability from fluorescence intensity [28]. DAPI and propidium iodide purchased from Sigma were used to detect cell viability. $\mathrm{IC}_{50}$ was calculated using an online tool: "Quest Graph ${ }^{\mathrm{TM}}$ IC50 Calculator." AAT Bioquest, Inc, https: / /www. aatbio.com/tools/ic50-calculator, accessed on 6 April 2021. The M-HeLa clone 11 human, epithelioid cervical carcinoma, strain of HeLa, clone of M-HeLa; human duodenal cancer cell line (HuTu 80) from the Type Culture Collection of the Institute of Cytology (Russian Academy of Sciences) and Chang liver cell line (Human liver cells) from the N. F. Gamaleya Research Center of Epidemiology and Microbiology were used in the experiments. The cells were cultured in a standard Eagle's nutrient medium manufactured at the Chumakov Institute of Poliomyelitis and Virus Encephalitis (PanEco company) and supplemented with $10 \%$ fetal calf serum and $1 \%$ nonessential amino acids. The cells were plated into a 96-well plate (Nanc) at a concentration of $1 \times 10^{5}$ cells $/ \mathrm{mL}, 150 \mu \mathrm{L}$ of medium per 
well, and cultured in a $\mathrm{CO}_{2}$ incubator at $37^{\circ} \mathrm{C}$. Twenty-four hours after seeding the cells into wells, the compound under study was added at a preset dilution, $150 \mu \mathrm{L}$ to each well. The dilutions of the compounds were prepared immediately in nutrient media; $5 \%$ DMSO, which does not induce inhibition of cells at this concentration, was added for better solubility. The experiments were repeated three times. Intact cells cultured in parallel with experimental cells were used as a control.

\subsection{Quantum Chemistry Calculations}

All calculations have been performed with the Gaussian 16 package [29]. The initial structures were fully optimized at the B3LYP $/ 6-311++G(d, p)$ theory level. All optimizations were followed by frequency calculations at the same level of theory in order to check that optimized structures really correspond to true minima.

\section{Conclusions}

In conclusion, a series of novel 4-(het) arylimidazolidines were obtained via trifluoroacetic acid catalyzed intramolecular cyclization/Mannich-type reaction of $\mathrm{N}-(2,2-$ diethoxyethyl) ureas with high regioselectivities. The plausible mechanism was discussed using quantum chemistry calculations and the choice of the solvent and catalyst amount was found to have a crucial effect on the regioselectivity. The anti-cancer activities of the obtained compounds were tested in vitro, and the substitution of the nitrogen atom was identified as one the key factors influencing the cytotoxicity against normal and cancer human cell lines.

Supplementary Materials: The following are available online. Figure S1: Bifurcate $\mathrm{CH} \ldots \mathrm{O}$ interaction in the crystals of the studied compounds, Table S1: CH ... O interactions in crystals of investigated compounds, Figures S2-S98: copies of the NMR spectra of all synthesized compounds.

Author Contributions: A.S.G., supervision (chemistry), conceptualization, writing and funding acquisition; A.V.S., E.A.K. and D.S.A., investigation (chemistry); A.R.B. and M.A.P., project administration; A.D.V., supervision (in vitro anti-cancer studies); V.V.S., NMR studies; A.P.L. and S.K.A., investigation (in vitro anti-cancer studies); J.K.V., investigation (X-ray studies and QC calculations). All authors have read and agreed to the published version of the manuscript.

Funding: The synthetic part of this work, the NMR studies and the biological studies were funded by the Russian Science Foundation, grant number 21-73-20020. The NMR studies were performed in the Assigned Spectral-Analytical Center of the FRC Kazan Scientific Center of RAS. The X-ray studies were supported by the Ministry of Science and Higher Education of Russia as part of the state assignment of the Kurnakov Institute of General and Inorganic Chemistry of the Russian Academy of Sciences.

Institutional Review Board Statement: Not applicable.

Informed Consent Statement: Not applicable.

Data Availability Statement: Not applicable.

Conflicts of Interest: The authors declare no conflict of interest.

Sample Availability: Samples of the compounds 2-7 are available from the authors.

\section{References}

1. Saibabu Kotti, S.R.S.; Timmons, C.; Li, G. Vicinal Diamino Functionalities as Privileged Structural Elements in Biologically Active Compounds and Exploitation of their Synthetic Chemistry. Chem. Biol. Drug Des. 2006, 67, 101-114. [CrossRef] [PubMed]

2. Lucet, D.; Le Gall, T.; Mioskowski, C. The Chemistry of Vicinal Diamines. Angew. Chemie Int. Ed. 1998, 37, 2580-2627. [CrossRef]

3. Smolobochkin, A.V.; Gazizov, A.S.; Burilov, A.R.; Pudovik, M.A.; Sinyashin, O.G. Advances in the synthesis of heterocycles with an endocyclic urea moiety. Russ. Chem. Rev. 2021, 90, 395-417. [CrossRef]

4. Wen, W.; Luo, M.-J.; Yuan, Y.; Liu, J.-H.; Wu, Z.-L.; Cai, T.; Wu, Z.-W.; Ouyang, Q.; Guo, Q.-X. Diastereodivergent chiral aldehyde catalysis for asymmetric 1,6-conjugated addition and Mannich reactions. Nat. Commun. 2020, 11, 5372. [CrossRef] [PubMed]

5. Zhou, M.; Li, K.; Chen, D.; Xu, R.; Xu, G.; Tang, W. Enantioselective Reductive Coupling of Imines Templated by Chiral Diboron. J. Am. Chem. Soc. 2020, 142, 10337-10342. [CrossRef] [PubMed] 
6. Li, J.; Zhang, D.; Chen, J.; Ma, C.; Hu, W. Enantioselective Synthesis of Fluoroalkyl-Substituted syn -Diamines by the Asymmetric gem -Difunctionalization of 2,2,2-Trifluorodiazoethane. ACS Catal. 2020, 10, 4559-4565. [CrossRef]

7. Du, M.; Yu, L.; Du, T.; Li, Z.; Luo, Y.; Meng, X.; Tian, Z.; Zheng, C.; Cao, W.; Zhao, G. N -Protecting group tuning of the enantioselectivity in Strecker reactions of trifluoromethyl ketimines to synthesize quaternary $\alpha$-trifluoromethyl amino nitriles by ion pair catalysis. Chem. Commun. 2020, 56, 1581-1584. [CrossRef]

8. Chen, Y.; Wu, Y.; Shatskiy, A.; Kan, Y.; Kärkäs, M.D.; Liu, J.-Q.; Wang, X.-S. Cooperative Silver- and Base-Catalyzed Diastereoselective Cycloaddition of Nitrones with Methylene Isocyanides: Access to 2-Imidazolinones. Eur. J. Org. Chem. 2020, 2020, 3475-3479. [CrossRef]

9. Tao, Z.; Gilbert, B.B.; Denmark, S.E. Catalytic, Enantioselective syn- Diamination of Alkenes. J. Am. Chem. Soc. 2019, 141, 19161-19170. [CrossRef]

10. Ivanovich, R.A.; Polat, D.E.; Beauchemin, A.M. Photocatalytic Intramolecular C-H Amination Using N -Oxyureas as Nitrene Precursors. Org. Lett. 2020, 22, 6360-6364. [CrossRef]

11. Zhou, Z.; Tan, Y.; Yamahira, T.; Ivlev, S.; Xie, X.; Riedel, R.; Hemming, M.; Kimura, M.; Meggers, E. Enantioselective Ring-Closing C-H Amination of Urea Derivatives. Chem 2020, 6, 2024-2034. [CrossRef]

12. Taily, I.M.; Saha, D.; Banerjee, P. Palladium-catalyzed regio- and stereoselective access to allyl ureas/carbamates: facile synthesis of imidazolidinones and oxazepinones. Org. Biomol. Chem. 2020, 18, 6564-6570. [CrossRef]

13. Sviridova, L.A.; Protopopova, P.S.; Akimov, M.G.; Dudina, M.S.; Melnikova, E.K.; Kochetkov, K.A. Synthesis of new physiologically active (2-oxoimidazolidin-5-yl)indoles. Mendeleev Commun. 2020, 30, 347-349. [CrossRef]

14. Gazizov, A.S.; Burilov, A.R.; Pudovik, M.A.; Sinyashin, O.G. Reactions of nitrogen-containing acetals with aromatic nucleophiles. Russ. Chem. Rev. 2017, 86, 75-98. [CrossRef]

15. Liu, D.-Y.; Zhu, S.-M.; Li, R.-Q.; Tian, J.-S.; Loh, T.-P. $\alpha$-Amino Acetal: A Synthetic Intermediate for the Construction of Aza-Polycycles. Org. Lett. 2019, 21, 6357-6360. [CrossRef] [PubMed]

16. Smolobochkin, A.; Gazizov, A.; Sazykina, M.; Akylbekov, N.; Chugunova, E.; Sazykin, I.; Gildebrant, A.; Voronina, J.; Burilov, A.; Karchava, S.; et al. Synthesis of Novel 2-(Het)arylpyrrolidine Derivatives and Evaluation of Their Anticancer and Anti-Biofilm Activity. Molecules 2019, 24, 3086. [CrossRef] [PubMed]

17. Smolobochkin, A.V.; Rizbayeva, T.S.; Gazizov, A.S.; Voronina, J.K.; Dobrynin, A.B.; Gildebrant, A.V.; Strelnik, A.G.; Sazykin, I.S.; Burilov, A.R.; Pudovik, M.A.; et al. Acid-Catalyzed Intramolecular Imination/Nucleophilic Trapping of 4-Aminobutanal Derivatives: One-Pot Access to 2-(Pyrazolyl)pyrrolidines. Eur. J. Org. Chem. 2019, 2019, 5709-5719. [CrossRef]

18. Smolobochkin, A.V.; Turmanov, R.A.; Abdullaeva, D.S.; Gazizov, A.S.; Voronina, J.K.; Appazov, N.O.; Buzyurova, D.N.; Burilov, A.R.; Pudovik, M.A. 2-(Het)aryl- N -phosphorylpyrrolidines via Cyclization of Phosphorus Acid Amides: A Regioselective Approach. ChemistrySelect 2020, 5, 12045-12050. [CrossRef]

19. Smolobochkin, A.V.; Gazizov, A.S.; Otegen, N.K.; Voronina, J.K.; Strelnik, A.G.; Samigullina, A.I.; Burilov, A.R.; Pudovik, M.A. Nucleophilic Cyclization/Electrophilic Substitution of (2,2-Dialkoxyethyl)ureas: Highly Regioselective Access to Novel 4-(Het)arylimidazolidinones and Benzo[d][1,3]diazepinones. Synthesis 2020, 52, 3263-3271. [CrossRef]

20. Gazizov, A.S.; Smolobochkin, A.V.; Burilov, A.R.; Pudovik, M.A. Interaction of 2-Naphthol with $\gamma$-Ureidoacetals. A New Method for the Synthesis of 2-Arylpyrrolidines. Chem. Heterocycl. Compd. 2014, 50, 707-714. [CrossRef]

21. Grant, H.M.; Mctigue, P.; Ward, D.G. The basicities of aliphatic amides. Aust. J. Chem. 1983, 36, 2211-2218. [CrossRef]

22. Chakraborty, S.; Saha, C. The Curtin-Hammett principle. Resonance 2016, 21, 151-171. [CrossRef]

23. Sheldrick, G.M. SHELXTL v.6.12, Structure Determination Software Suite; Bruker AXS: Madison, WI, USA, 2000.

24. Dolomanov, O.V.; Bourhis, L.J.; Gildea, R.J.; Howard, J.A.K.; Puschmann, H. OLEX2: A complete structure solution, refinement and analysis program. J. Appl. Crystallogr. 2009, 42, 339-341. [CrossRef]

25. Llopart, C.C.; Ferrer, C.; Joule, J.A. Lithiation of 1-arylimidazol-2(1 H )-ones and 1-aryl-4,5-dihydroimidazol-2(1 H )-ones. Can. J. Chem. 2004, 82, 1649-1661. [CrossRef]

26. Hu, Y.-C.; Liang, C.-F.; Tsai, J.-H.; Yap, G.P.A.; Chang, Y.-T.; Ong, T.-G. Zirconium Complexes Supported by Imidazolones: Synthesis, Characterization, and Application of Precatalysts for the Hydroamination of Aminoalkenes. Organometallics 2010, 29, 3357-3361. [CrossRef]

27. Burilov, A.R.; Khakimov, M.S.; Gazizov, A.S.; Pudovik, M.A.; Syakaev, V.V.; Krivolapov, D.B.; Konovalov, A.I. Reactions of resorcinol derivatives with 1-methyl-3-phenylimidazol-2-one as a new method for the synthesis of 5-arylimidazolidin-2-ones. Mendeleev Commun. 2008, 18, 54-55. [CrossRef]

28. Voloshina, A.D.; Sapunova, A.S.; Kulik, N.V.; Belenok, M.G.; Strobykina, I.Y.; Lyubina, A.P.; Gumerova, S.K.; Kataev, V.E. Antimicrobial and cytotoxic effects of ammonium derivatives of diterpenoids steviol and isosteviol. Bioorgan. Med. Chem. 2021, 32, 115974. [CrossRef] [PubMed]

29. Frisch, M.J.; Trucks, G.W.; Schlegel, H.B.; Scuseria, G.E.; Robb, M.A.; Cheeseman, J.R.; Scalmani, G.; Barone, V.; Petersson, G.A.; Nakatsuji, H.; et al. Gaussian 16, Revision C.01; Gaussian, Inc.: Wallingford, CT, USA, 2016. 